\title{
Systems Pharmacology Dissection of Multi-Scale Synergistic Treatment Strategies of Chaihu Shugan Powder for Depression
}

\author{
Yu-Jing Zhao',2, Feng Li ${ }^{2,3}$, Qi-Lei Liü, Yan Li ${ }^{1^{*}}$ \\ ${ }^{1}$ Key Laboratory of Industrial Ecology and Environmental Engineering, Faculty of Chemical, Environmental and \\ Biological Science and Technology, Dalian University of Technology, China \\ ${ }^{2}$ Henan University of Engineering, China \\ ${ }^{3}$ University Malaysia Sarawak (UNIMAS), Malaysia \\ ${ }^{4}$ Institute of Chemical Process Systems Engineering, School of Chemical Engineering, Dalian University of \\ Technology, China
}

\section{OPEN ACCESS}

${ }^{*}$ Correspondence:

Yan Li, Key Laboratory of Industrial

Ecology and Environmental

Engineering, Environmental and

Biological Science and Technology,

Dalian University of Technology, Dalian

116024, China,

E-mail: yanli@dlut.edu.cn

Received Date: 18 Oct 2019

Accepted Date: 01 Nov 2019

Published Date: 18 Nov 2019

Citation:

Yu-Jing Zhao, Feng Li, Qi-Lei Liu, Yan Li. Systems Pharmacology Dissection of Multi-Scale Synergistic Treatment Strategies of Chaihu Shugan Powder

for Depression. J Gynecol Oncol. 2019; 2(3): 1020

Copyright $\odot 2019$ Yan Li. This is an open access article distributed under

the Creative Commons Attribution

License, which permits unrestricted use, distribution, and reproduction in any medium, provided the original work is properly cited.

\begin{abstract}
Depression has a high morbidity and mortality worldwide, and because it is often accompanied by a series of complications, drugs that target a single protein or pathway may not fully exerts desired therapeutic effect. Traditional Chinese Medicine (TCM) plays a significant therapeutic effect in the treatment of depression and has fewer adverse reactions. However, taking a representative formula Chaihu Shugan Powder (CSP) as an example, which shows pharmacological effects on depression and its synergistic mechanism, remains still ambiguous. Our study aims to provide a holistic perspective of the correlation between TCM formula and depression, and to explore the synergistic mechanisms of CSP for the treatment of depression from multiple scales. In this study, we carry out systems pharmacology study on CSP by applying integrated of ADME evaluation, target fishing, protein-protein interaction analysis, gene ontology enrichment analysis, establishment of a directed network and network pharmacology analysis. As a result, 126 chemicals and 83 proteins are identified as active compounds and depression-related targets, and the synergistic effects of CSP formula for the treatment of depression are based on its three mechanisms of anti-inflammation, and the regulation of neuroendocrine system and monoamine neurotransmitter through the smooth operation of complex biological pathways, including especially PI3K-Akt, cAMP and MAPK signaling pathways. Moreover, network analysis reveals that CSP is also capable of relieving or even certainly eliminating side effects and complications of depression due to the abundant active ingredients in CSP formula.
\end{abstract}

Conclusion: This work not only provides insights into the synergy of TCM for depression treatment, but also provides a new and effective way to develop potential antidepressants and targets.

Keywords: Depression; Systems pharmacology; Traditional Chinese Medicine; Chaihu Shugan Powder; Synergistic mechanism; Antidepressants

\section{Abbreviations}

5-HT: Serotonin; AC: Adenylate Cyclase; ACTH: Adrenocorticotropic Hormone; ADME: Absorption Distribution Metabolism and Excretion; AR: Androgen Receptor; AVP: Arginine Vasopressin; BS: Radix Paeoniae Alba; CH: Radix Bupleuri; COX: Cyclooxygenase; CP: Citrus Reticulata; CRH: Corticotropin Releasing Hormone; CSP: Chaihu Shugan Powder; C-T: Compound-Target; C-T-D: Compound-Target-Disease; C-T-P: Compound-Target-Pathway; CX: Rhizoma Chuanxiong; DA: Dopamine; DL: Drug-Likeness; DRD1: D (1A) Dopamine Receptor; D-T: Drug-Target; E2: Estrogen Estradiol; ESR1: Estrogen Receptor a; FDR: False Discovery Rate; GC: Glucocorticoid; GC: Licorice; GO: Gene Ontology; GR or NR3C1: Glucocorticoid Receptor; GSK3B: Glycogen Synthase Kinase-3ß; HL: Half-Life; HPA: Hypothalamic-Pituitary-Adrenal; IL: Interleukin; Inf-C-T: Inflammation-Compound-Target; KEGG: Kyoto Encyclopedia of Genes and Genomes; MAOIs: Monoamine Oxidase Inhibitors; MAPK14: p38 Mitogen-Activated Protein Kinase; MDD: Major Depression Disorder; Mon-C-T: Monoamine Neurotransmitter-CompoundTarget; MR or NR3C2: Mineralocorticoid Receptor; NaRIs: Noradrenaline Reuptake Inhibitors; NE: Noradrenaline; Neu-C-T: Neuroendocrine-Compound-Target; NRIs: NE Reuptake Inhibitors; OB: 
Oral Bioavailability; PG: Prostaglandin; PGE2: Prostaglandin E2; PGR: Progesterone Receptor; PPARs: Peroxisome Proliferator-Activated Receptors; PPI: Protein-Protein Interaction; PTGS2: Prostaglandin H Synthase 2; PVN: Paraventricular Nucleus; RF: Random Forest; SERT: Serotonin Transporter; SP: Systems Pharmacology; SSRIs: Selective Serotonin Reuptake Inhibitors; SVM: Support Vector Machine; SysDT: Systematic Drug Targeting Tool; TCAs: Tricyclic Antidepressants; TCM: Traditional Chinese Medicine; TCMSP: Traditional Chinese Medicine Systems Pharmacology; TNF- $\alpha$ : Tumor Necrosis Factor- $\alpha$; T-P: Target-Pathway; TST: Tail Suspension Test; XF: Rhizoma Cyperi; ZK: Fructus Aurantii; $\alpha 1$-ARs: Adrenergic $\alpha 1$ Receptors; $\beta$-ARs: Adrenergic $\beta$ Receptors

\section{Introduction}

Depression typically refers to unipolar depression, also known as clinical or Major Depression Disorder (MDD) [1], which is characterized by three core symptoms: low mood, anhedonia and low energy levels, as well as cognitive impairment, sleep disorders, sexual dysfunction and gastrointestinal diseases, etc. accompanying symptoms. Although depression is not an incurable disease, the combination of the primary and the secondary disabilities of chronic medical illness it causes makes MDD one of the most costly medical burdens in the world [2]. Actually, who has predicted that MDD will become the second leading cause of disability worldwide by the year 2030 [3]. Therefore, the study on depression therapy is quite a hot field which has attracted widespread attention. In the 1950s, scientists stumbled upon the discovery of the capability of anti-tuberculosis drugs (iproniazid) and tricyclic compounds (imipramine) in improving people's mood and effectively treating depression. Based on these fundamental chemicals, subsequently, commercially available clinically used antidepressants were gradually developed [4]. The first-generation antidepressants include Monoamine Oxidase Inhibitors (MAOIs) and Tricyclic Antidepressants (TCAs) [5], both of which elevate extracellular monoamine levels by either blocking Monoamine Oxidase (MAO) (like iproniazid) or inhibiting the neuronal Serotonin and Noradrenaline transporter (5-HT/NE) (like imipramine and its active metabolite desipramine [4]), and thereby exert antidepressant effects. Although these antidepressants exhibit the longest record of use in clinical and a wide antidepressant spectrum, with a total therapeutic efficiency of $70 \%$ to $80 \%$ in all types of depression, they often possess certain undesirable side effects and toxic effects in overdose, which greatly limit their application [5,6]. Therefore, by the 1980s, newer-generation antidepressants were developed, including Selective Serotonin Reuptake Inhibitors (SSRIs) and Noradrenaline Reuptake Inhibitors (NaRIs), Serotonin Noradrenergic Reuptake Inhibitors (SNaRIs), Noradrenergic and Specific Serotonergic Antidepressants (NaSSAs), Serotonin Reuptake Inhibitor (SRIs), etc. [6]. Compared with the first-generation, these antidepressants are more selective and offer improved safety and tolerability [5]. However, they are less effective than the firstgeneration of antidepressants that the existing drug therapy is only suitable for the treatment of depression in $60 \%$ to $70 \%$ of patients. In addition, there is a slower onset of action for these two types of antidepressants. It takes an average of 6 weeks to 7 weeks for depression patients to recover from health, which is usually accompanied by a high risk of suicide, so slow onset is another cause of life threatening. Therefore, efforts to find more effective antidepressants are still research hotspots.

Traditional Chinese Medicine (TCM) is complex mixtures that contain a variety of ingredients acting on multiple targets and pathways to systematically treat diseases. TCM, as the main therapy of complementary and alternative medicine, has a long history of more than 1,000 years [7]. TCM also has rich clinical experience in the treatment of depression, with specific advantages in regulation of complications of depression such as gastrointestinal disorders and sexual dysfunction with fewer adverse reactions [6]. It has been widely applied in depressive treatment in recent decades. For example, in Taiwan, more than $40 \%$ of depressed patients used TCM services. Growing preclinical and clinical trials also show beneficial efficacy of TCM in the treatment of depression [7]. Among them, Chaihu Shugan Powder (CSP) formula has achieved remarkable results in the treatment of depression [8-10]. For example, Sun Yan et al. [11] systematically searched several databases for randomized controlled trials of CSP in depression, which compared CSP (or combined with a conventional Western medicine) with a regular Western medicine alone in the treatment of depression. They have included 42 studies involving 3234 patients with depression in 15 different types of diseases. Meta-analysis showed that CSP had better efficacy than fluoxetine for pure depression, for post-stroke depression and for postpartum depression. No article has reported serious oral adverse events in CSP. In addition, any adverse reactions using CSP alone were less than conventional Western medicines [11]. Therefore, the development of TCM with fewer side effects from natural herbs is very promising. Unlike Western medicine, TCM is typically characterized by multiple components and multiple targets as well as complex pharmacological mechanisms. Therefore, it is exceedingly difficult to explore the pathological mechanism of depression using only traditional experimental methods. Fortunately, Systems Pharmacology (SP) provides a new perspective. It is a new inters discipline developed based on classical pharmacology, chemical biology, applied mathematics, computer technology and bioinformatics, resembling the holistic concept of TCM channel orientation. Recently, growing bodies of studies have demonstrated the successful applications of SP in systematically investigation and elucidation of the scientific connotation of Chinese medicine theory, in analysis of the mechanism of TCM herbs for treating diseases, as well as in providing powerful technical supports for laboratory drug discovery derived from TCM herbs [12-14]. Therefore, this work utilizes a SP strategy to investigate the synergy mechanism of CSP in depression treatment from the four-scale perspectives of molecule, target, network and pathway.

\section{Materials and Methods}

\section{Molecular database building and active ingredients screening}

All chemicals of CSP were obtained from Traditional Chinese Medicine Systems Pharmacology (TCMSP) database (http://lsp.nwu. edu.cn) and literature search [15]. Then, three in silico, including Oral Bioavailability (OB), predict Half-Life (HL) and Drug-Likeness (DL) ADME (Absorption, Distribution, Metabolism and Excretion) models were applied for filtering out the potentially active molecules of CSP [16-18]. The threshold values for the screening models were $\mathrm{OB} \geq 30 \%, \mathrm{DL} \geq 0.18$, and $\mathrm{HL} \geq 4$, respectively [9]. The ingredients which successfully satisfy all the criteria were kept as candidate compounds for further analysis.

\section{Target fishing}

In this work, the candidate targets of CSP were forecasted by Systematic Drug Targeting tool (SysDT) algorithm, which was 


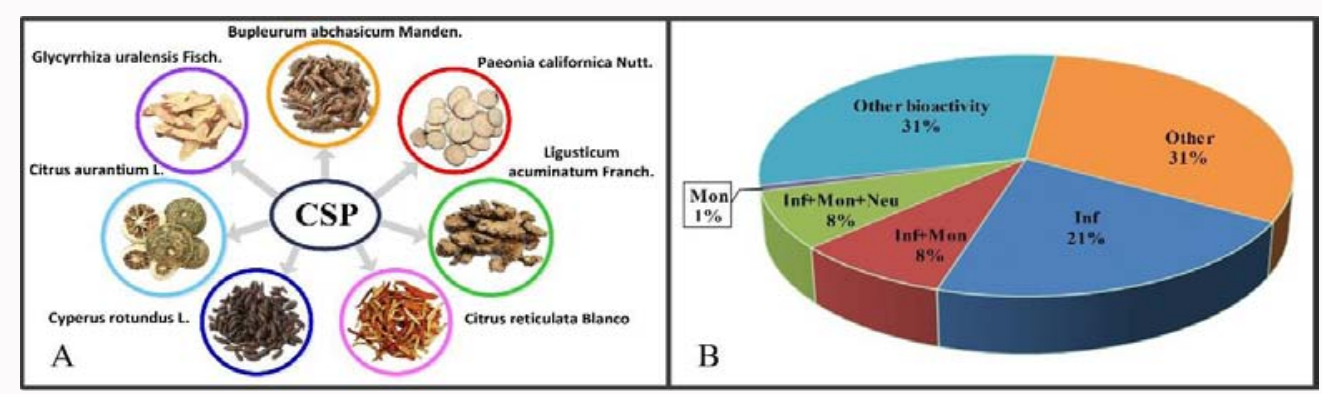

Figure 1: A) Herbs of CSP formula B) and the classification of the bioactive ingredients of CSP according to their pharmacological functions.

developed based on combinational use of two mathematical tools: Random Forest (RF) and Support Vector Machine (SVM). SysDT was applied to identify those potential targets (using RF score $\geq$ 0.8 and $\mathrm{SVM} \geq 0.7$ as threshold). The resulted targets were then mapped to UniProt database (http://www.uniprot.org/) to achieve normalization of the targets' writing form.

\section{Protein-protein interaction analysis}

In order to further confirm whether the above resulted targets were closely related to depression, the most commonly used clinical antidepressants with their corresponding targets were then collected through text mining and Drug Bank database searching. Thereafter, targets of both CSP and the antidepressants were mapped to STRING database (https://string-db.org/) to obtain corresponding human Protein-Protein Interaction (PPI) data. Later, a PPI analysis was conducted with purpose to analyze the relationships among all these targets. The degree of correlation between CSP and antidepressants' targets was evaluated using the measurement of shortest path length, as calculated by (Equation 1) [19]:

$$
d_{s}(S, T)=\frac{1}{\|T\|} \sum_{t \in T} \frac{1}{\|S\|} \sum_{s \in S} d(s, t)
$$

Where $S$ and $T$ are the sets of antidepressant targets and CSP targets, and $d_{s}(S, T)$ is the shortest path length between nodes $s$ and $t$ in the PPI network, respectively.

To evaluate the significance of distances between CSP and antidepressants' targets, a reference distance distribution was generated by calculating the proximity between two randomly selected groups repeating 1,000 times [19]. The proximity is defined in (Equation 2):

$$
z(S, T)=\frac{d(S, T)-\mu_{d(S, T)}}{\sigma_{d(S, T)}}
$$

Where an observed distance is converted to a normalized distance using the Mean $\mu_{d(S, T)}$ and SD. $\sigma_{d(S, T)}$ of the reference distribution.

\section{Gene ontology enrichment analysis}

To further explore what vital biological processes that CSP targets are involved in, a Gene Ontology (GO) enrichment analysis was performed by mapping the targets to DAVID database (http://david. abcc.ncifcrf.gov) [20]. Then, to better illustrate the resulted biological processes, those GO terms with $\mathrm{p}$-value $\leq 0.05$ were screened out for further study.

\section{PreAM: predict mode of action for drugs}

To rigorously assess the pharmacological effects of the active ingredient and the corresponding target, another internal tool,
preAM, based on a robust random forest algorithm, is used to determine drug-target interactions in two modes: activation and inhibition.

\section{Network construction and analysis}

In this section, Compound-Target (C-T), Target-Pathway ( $\mathrm{T}$ $\mathrm{P})$ and Drug-Target (D-T) visualized networks were constructed (using Cytoscape 3.6.0 software). In general, compounds, targets and pathways were represented by nodes while edges indicate the interactions among them, and the fundamental topological properties of these networks were analyzed to reveal the multi-target and multimechanisms therapeutic feature of CSP formula using Cytoscape's web analytics plugin.

\section{Pathway constructions and analysis}

To delve into the mechanisms that how targets affect the diseases via Specific pathways, all depression-related pathways were integrated based on the current cognition of depression pathology. Briefly, all CSP targets were firstly input into the Kyoto Encyclopedia of Genes and Genomes (KEGG) database (https://www.kegg.jp/) to extract the necessary information of pathways $[21,22]$. Subsequently, the enriched pathways of targets were examined with the evaluation of False Discovery Rate (FDR) less than 0.05 through Fisher's exact test in DAVID database $(F D R<0.05)$ [20]. Finally, a relatively complete depression-related pathway was established for further analysis of the molecular synergy mechanisms.

\section{Results}

\section{Revealing the synergistic action from component level}

Initially recorded in Jing Yue Quan Shu. Ancient Eight Arrays four hundred years ago, CSP is composed of seven herbs: Bupleurum abchasicum Manden. (pinyin abbreviation is $\mathrm{CH}$ ), Paeonia californica Nutt. (BS), Ligusticum acuminatum Franch. (CX), Citrus reticulata Blanco (CP), Cyperus Rotundus L. (XF), Citrus aurantium L. (ZK), and Glycyrrhiza uralensis Fisch. (GC) see in (Figure 1A). All herbal names correspond to the latest revision in "The Plant List" (www. theplantlist.org) or MPNS (http://mpns.kew.org). Despite of the great therapeutic effects that CSP exhibits in clinical treatment of depression, the identification of its active ingredients in treating depression is really difficult, due to the fact that it's each herb contains dozens or even hundreds of components. Thus, to better explore its therapeutic mechanism, in the present work, a CSP ingredient database was firstly established through data mining method, which ended up with a component pool composed of 1,095 ingredients in total with diversified structures. Considering that CSP is orally administrated in clinic, it will function efficiently only when its bioactive ingredients reach targets with appropriate concentrations. Thus, the identification of those components with satisfactory 
Table 1: The herbs of CSP formula.

\begin{tabular}{|c|c|c|c|c|c|}
\hline \multirow{2}{*}{ No. } & \multirow{2}{*}{ Latin name } & \multirow{2}{*}{ Chinese pinyin } & \multicolumn{2}{|c|}{ Number } & \multirow[t]{2}{*}{ Abb. } \\
\hline & & & Ingredients & Candidates & \\
\hline 1 & Bupleurum abchasicum Manden. & Chaihu & 349 & 16 & $\mathrm{CH}$ \\
\hline 2 & Paeonia californica Nutt. & Baishao & 87 & 12 & BS \\
\hline 3 & Ligusticum acuminatum Franch. & Chuanxiong & 190 & 10 & $\mathrm{CX}$ \\
\hline 4 & Citrus reticulata Blanco & Chenpi & 63 & 7 & $\mathrm{CP}$ \\
\hline 5 & Cyperus rotundus L. & Xiangfu & 104 & 15 & $X F$ \\
\hline 6 & Citrus aurantium L. & Zhike & 17 & 5 & ZK \\
\hline 7 & Glycyrrhiza uralensis Fisch. & Gancao & 285 & 84 & GC \\
\hline
\end{tabular}

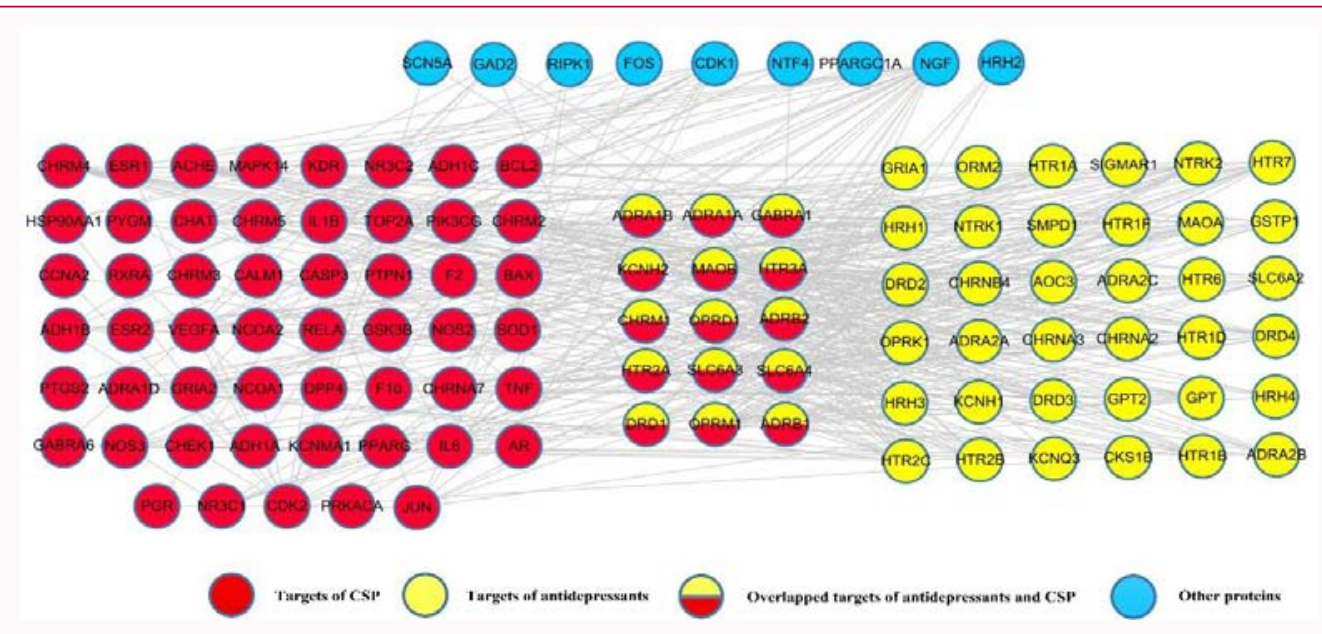

Figure 2: PPI network, where the yellow nodes represent the targets of clinically used antidepressants, the red nodes represent potential CSP targets, the nodes of half red and half yellow are the overlapped targets of clinical drugs and CSP, and the blue nodes are other proteins that interact with both the antidepressants' targets and CSP targets, respectively.

pharmacokinetic properties is the first key step for exploring the action mechanisms of CSP. Therefore, an ADME property screening platform that consists of $\mathrm{OB}, \mathrm{DL}$, and HL modules was applied on CSP to identify all potential active ingredients. Among them, $\mathrm{OB}$ represents the percentage of oral administration of a given compound that is administered to the body to circulate to produce a pharmacological effect in organisms [16]. DL index refers to whether the expected compound has physical and chemical properties and structural characteristics consistent with most known drugs, which is applied for identifying molecules with "drug like" characteristics [23]. $\mathrm{HL}$ is defined as the time required for a compound in the body to decrease to half its initial value. They are all important considerations for guiding the design and selection of molecules in early stages of drug discovery and development. In addition, several ingredients such as glycyrrhizic acid and $18 \beta$-glycyrrhetic acid are also treated as candidates due to their abundance in CSP herbs. In this way, finally, a total of 126 candidate compounds were obtained listed in (Supplementary Table A1). Also, CSP herbs' name, the number of ingredients they contain and corresponding abbreviations are shown in Table 1 .

An interesting phenomenon is observed that more than half of CSP candidate compounds (69\%) are biologically active in various experiments, as shown in Figure 1B. Specifically, among all bioactive molecules, 37\% have anti-inflammatory functions. Like glabridin (C157), a GC ingredient, significantly inhibits the release of both NO andinterleukin-1 $\beta$ (IL-1 $\beta$ ), as well as the production of both prostaglandin E2 (PGE2) and leukotriene B $[24,25]$. In addition,
$17 \%$ of the candidate compounds participate in the regulation of monoamine neurotransmitter. Isoliquiritin (C200) is a good example which significantly reduces the ratio of 5-HIAA/5-HT in the hippocampus, hypothalamus and cortex, slowing down 5-HT metabolism compared with mice treated with vehicle + stress [26]. Besides, $8 \%$ have functions of regulating neuroendocrine system. E.g. baicalin (H071) normalizes the function of Glucocorticoid Receptor (GR) through SGK1 and FKBP5-mediated GR phosphorylation, and thus improves anxiety/depression-like behavior [12]. As to the rest, $31 \%$, they also possess certain functions like neuroprotection. For example, senkyunolide A (X186) regulates PP2A activity and $\alpha$-syn levels in corticosterone-induced depression cell model, and in this way exerts neuroprotective effects [27]. To sum up, the biological functions of CSP components vary from each other, indicating their complementary pharmacological roles in treating depression. Candidate compounds in CSP play a synergistic effect in depression treatment through this complementary or mutually promoted effect. The CSP "multi-compound" property helps to identify the three functions of anti-inflammation, adjusting the neuroendocrine system and monoamine neurotransmitters that are strongly associated with antidepressant therapy. This also indicates the potential therapeutic mechanisms in the treatment of depression.

\section{Revealing the pharmacological synergy from target level}

Active ingredients generally act on certain proteins to exert their bioactivities. The identification of these proteins is thus essential for elucidating CSP antidepressant mechanisms. Therefore, presently, target fishing was carried out, which results in 83 proteins being 


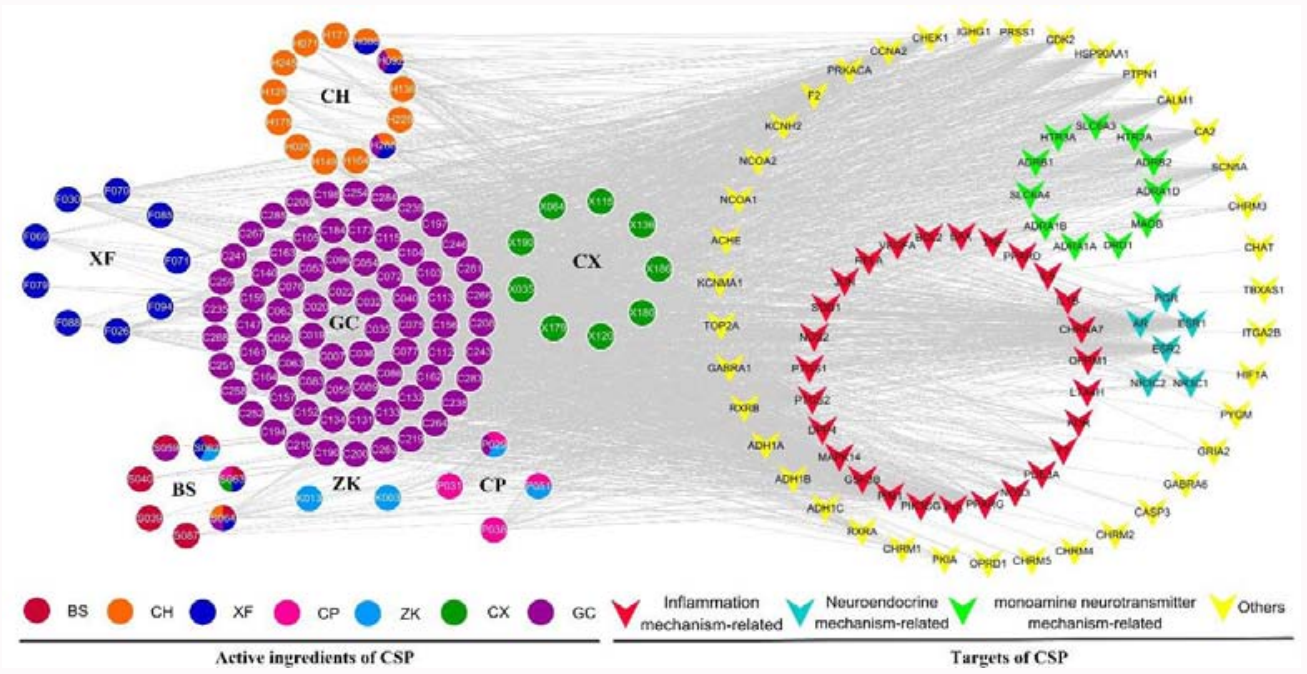

Figure 3: C-T network of CSP formula, in which the circles and arrows represent the biologically active ingredients and targets of CSP, respectively. Among the targets, red, blue, and green arrows represent inflammation-, neuroendocrine-, and monoamine neurotransmitters- related proteins, respectively.
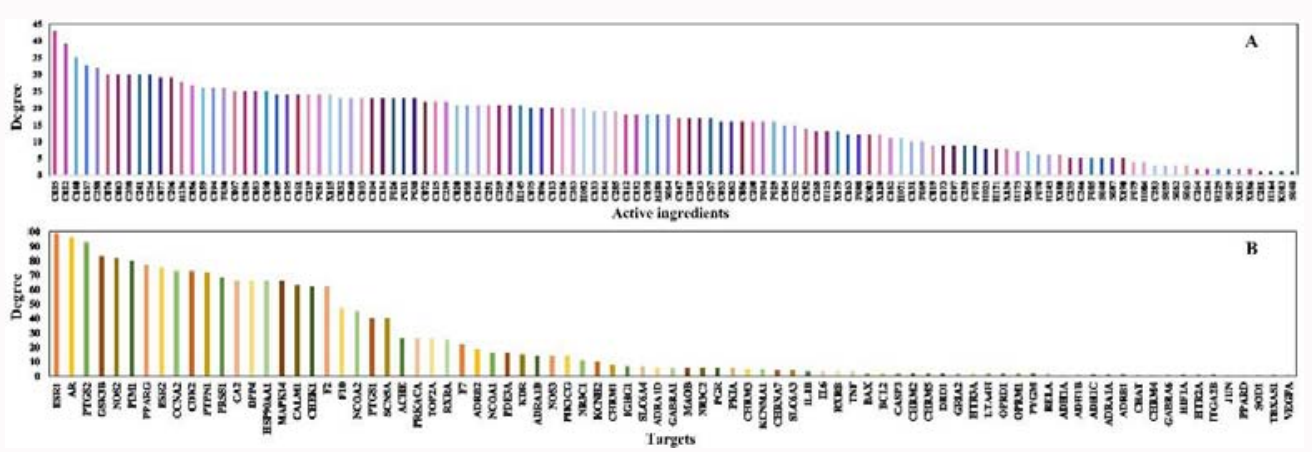

Figure 4: A) The degree distribution of active ingredients B) and targets of CSP formula.

identified as CSP target ( $T$ set) (Supplementary Table A2).

Then, all current clinically widely used antidepressants were collected, which results in 26 antidepressants with corresponding 54 targets (Supplementary Table A3). And all these 54 targets were put into $S$ set.

To measure the relationship of the above two sets of targets, a Protein-Protein Interactions (PPI) analysis was carried out here, where the shortest path length $d_{s}(S, T)$ between sets $S$ and $T$ was calculated by a nearness analysis. As for $d_{s}$, its corresponding relative proximity $z$ captures the statistical significance (z-score) of the observed targetdisease protein distance compared with the respective random expectation. As a result, the proximity $(z(S, T))$ between the two sets is smaller than -0.15 , which is considered highly proximal [19]. And $\left(z(S, T)=-5.304\left(d_{s}(S, T)=3.14\right.\right.$ and $p=0.003$, indicating that the two sets of targets are highly correlated with each other. To further identify the direct/indirect relationships among these proteins, a comprehensive PPI network is then built, as shown in Figure 2.

Interestingly, it is observed that 15 CSP targets are also clinical antidepressants' targets, which have been proved to have great treatment effects on depression. For example, $M A O B$ is a well-known target for antidepressants and neuroprotective drugs, SLC6A4 is the main target in most SSRI antidepressant genetics studies [28], HTR2A and HTR3A have been implicated in the aetiology and treatment of affective disorders such as anxiety and depression, $S L C 6 A 3$ is a susceptibility gene for depression $[29,30]$. This fact indicates that the anti-depression efficacy of CSP attributes a lot to a mechanism similar to current $\mathrm{ADs}$ by directly interacting with these overlapped targets.

In addition, some specific targets of CSP also contribute significantly to its treatment of depression. Among them, there are two aspects of influence. On the one hand, some CSP targets act directly on clinical drug targets. E.g. both DRD2 and MAOA, two clinical antidepressants targets, directly interact with 17 CSP targets, and similarly, 16 CSP targets also impact HTR2C in a direct way. On the other hand, some CSP targets may also indirectly affect certain antidepressants targets through a third protein. For instance, through SCN5A, CDK2, a CSP target, also influences KCNH1, an antidepressant target. These results reflect that the antidepressant efficacy of CSP depends on greatly the direct or indirect interactions of its active ingredients on clinical antidepressants' targets.

\section{The synergy analysis from network level}

To further investigate the synergistic mechanisms of CSP on depression treatment, here, a C-T network was generated by using all candidate compounds and potential targets of CSP, which is composed of 203 nodes and 1,983 lines, as shown in Figure 3.

Since degree refers to a fundamental topological parameter of a network, the number of edges the node connects with. Its calculation helps to quantify the node importance so as to identify the most influential nodes in networks. The degree distribution of CSP targets 


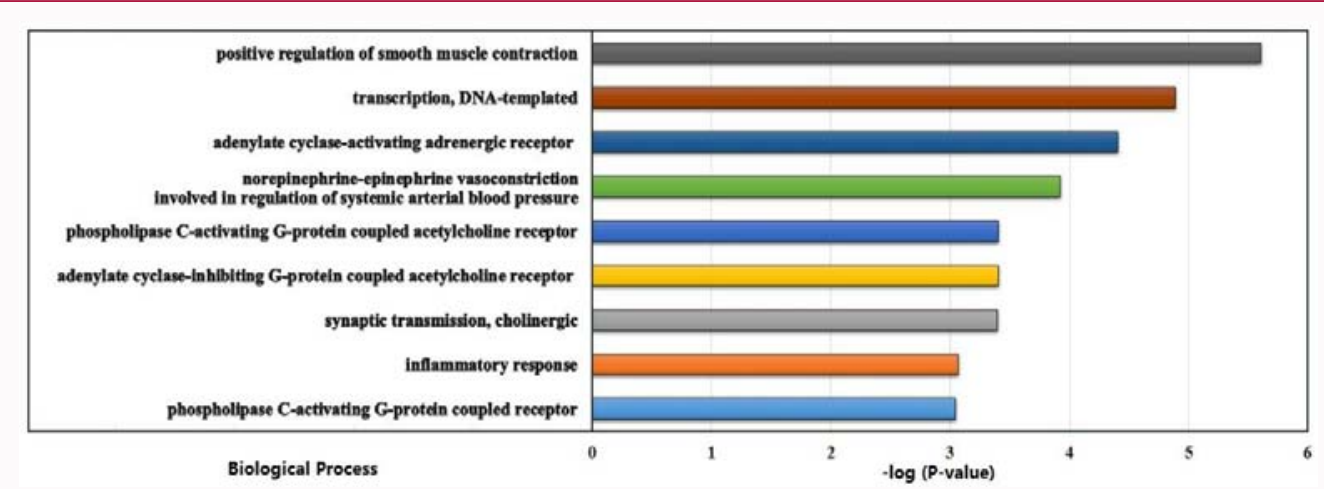

Figure 5: Gene Ontology analysis of the target genes, where y-axis is the significantly enriched 'Biological Process' categories in GO relative to the target genes, and $\mathrm{x}$-axis is the enrichment scores of these terms $p \leq 0.05$.

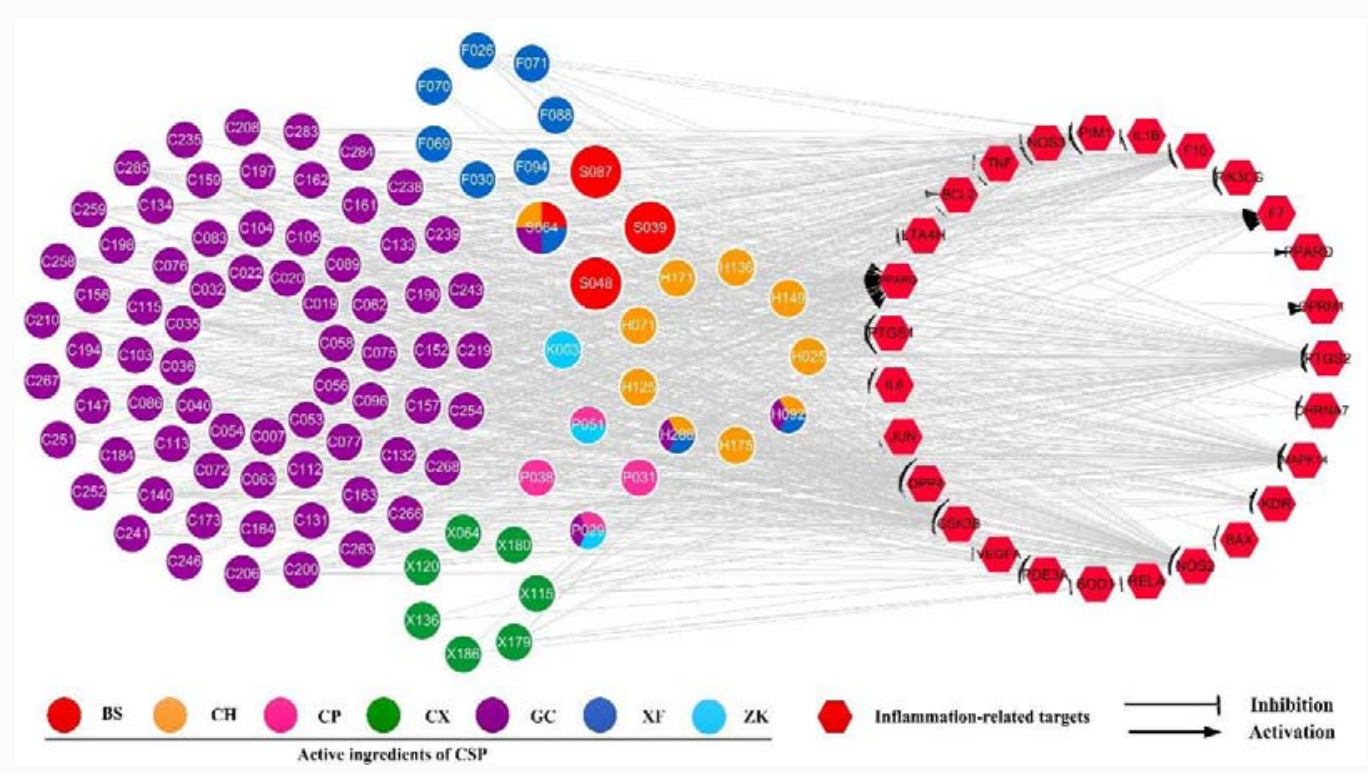

Figure 6: Inf-C-T network of CSP formula, where the hexagons and circles represent all inflammation-related CSP targets and their corresponding active ingredients, respectively. The grey edge represents the interrelationship between the target and the chemical. The node size is proportional to its degree.

and active compounds Shown in Figure 4. For CSP targets, their average degree (i.e. the average number of candidate compounds attached to each target) is 24 , and $82 \%$ targets interact with at least two or more candidate compounds (degree $\geq 2$ ), which indicates that the therapeutic effect of most proteins results from a variety of candidate compound interactions and also CSP shows a cumulative drug effect in the treatment of depression. Since undoubtedly, more compounds a protein interacts with, more critical the protein is [31]. Those high-degree nodes like the top three targets, i.e., Estrogen Receptor a (ESR1), Androgen Receptor (AR) and Prostaglandin $\mathrm{H}$ Synthase 2 (PTGS2), make the greatest impact on CSP curative effects. In addition, the mean degree of CSP active compounds, i.e. the mean number of targets they interact, is 16 , demonstrating the poly pharmacology property and "multi-target" characteristics of CSP constituents. Actually, 53 out of all active ingredients (40\%) interact with more than 20 proteins, also proving this characteristic as well as their crucial roles.

Gene Ontology (GO) enrichment analysis: As observed in the C-T network, more than half of CSP targets have antiinflammation function, and/or regulation of neuroendocrine system and of monoamine neurotransmitters; therefore, CSP may treat depression through these three functions (Figure 3). To validate this assumption, a GO enrichment analysis was performed here using all CSP targets. The results show that these targets are involved in dozens of biological processes that are associated with the pathogenesis of depression (Figure 5). More specifically, most of the highly enriched GO terms are tightly related to inflammation, neuroendocrine and monoamine neurotransmitter. For example, adenylate cyclase-inhibiting G-protein coupled acetylcholine receptor and phospholipase C-activating G-protein coupled receptor are both closely in connection with neuroendocrine, while synaptic transmission and adenylate cyclase-activating adrenergic receptor are associated with monoamine neurotransmitter. This is very consistent with the pathogenesis of depression which is usually caused by three major physiological mechanisms, namely inflammation, neuroendocrine, and monoamine neurotransmitter imbalance. Thus, CSP may work through these three mechanisms in the treatment of depression. To address this, three C-T networks, i.e. Inf-C-T (Inflammation-Compound-Target) network, NeuC-T (Neuroendocrine-Compound-Target) network and Mon-C-T (Monoamine Neurotransmitter-Compound-Target) network, are further constructed respectively, with details analyzed as follows. 

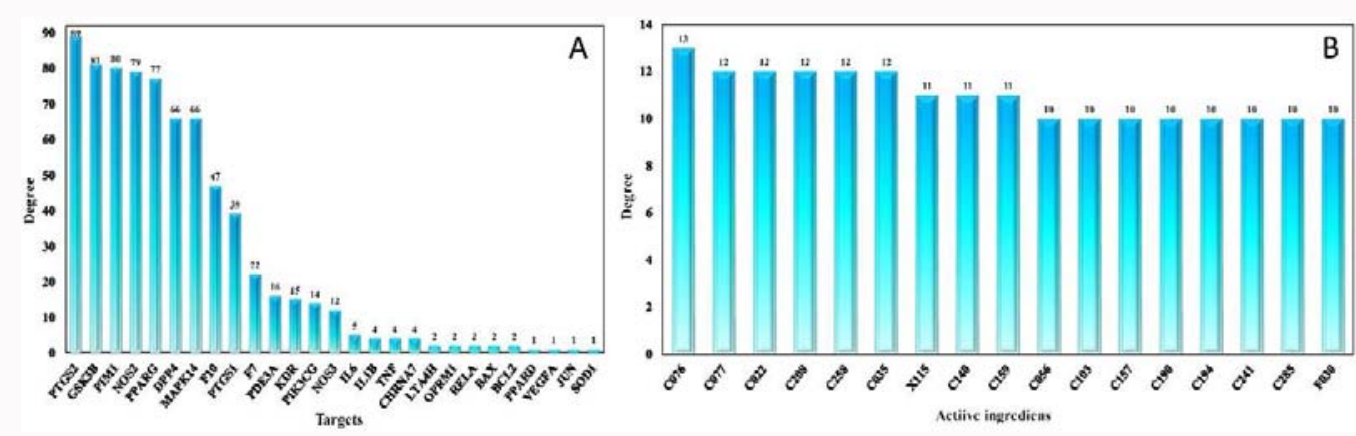

Figure 7: A) The degree distribution of the targets $B$ ) and those highly-degreed (with degree value $\geq 12$ ) active ingredients of CSP formula in the Inf-C-T network.

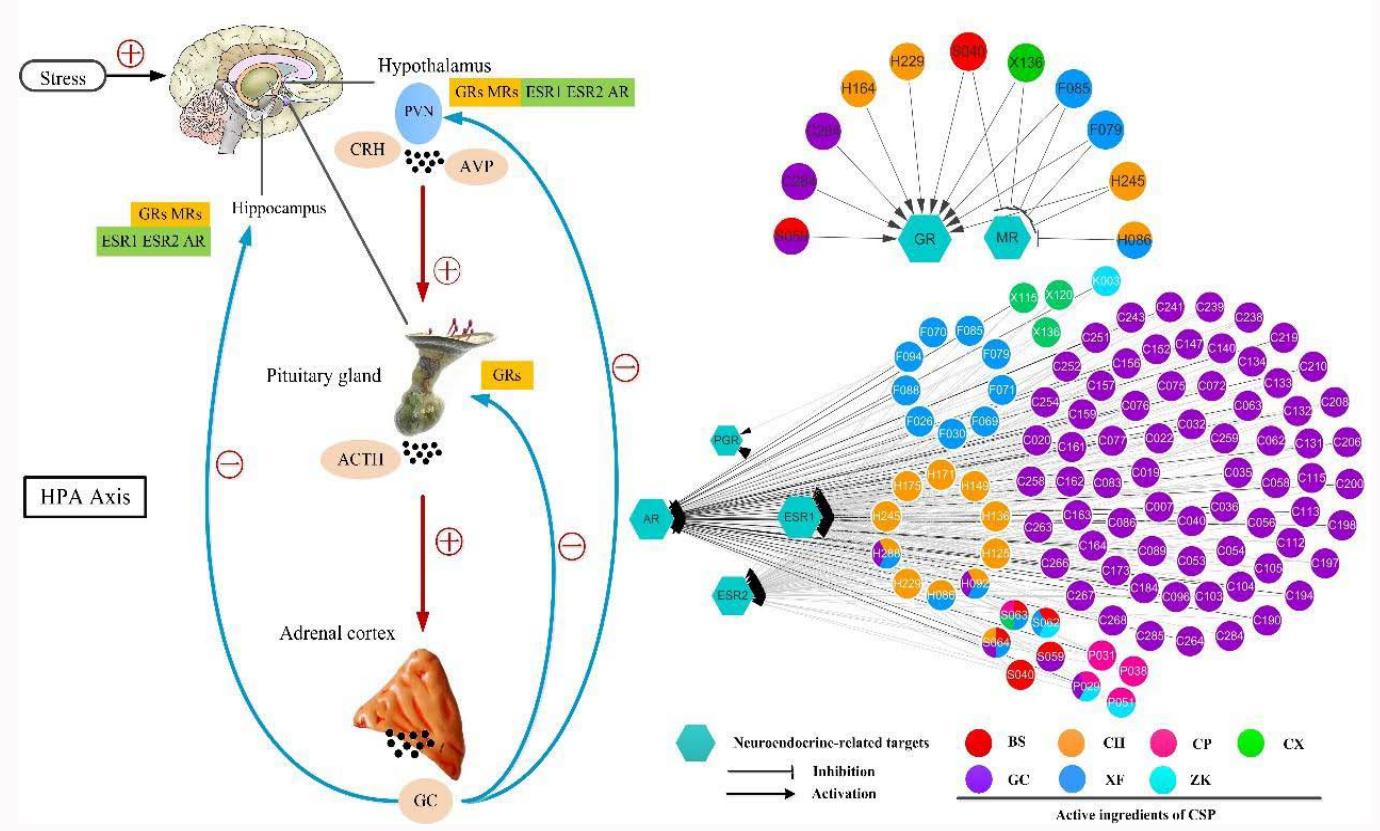

Figure 8: Neu-C-T network of CSP formula, where the hexagons and circles represent all neuroendocrine-associated CSP targets and corresponding active ingredients, respectively. The grey edge represents the interrelationship between the target and the chemical. The node size is proportional to its degree.

Inflammation mechanism: Chronic stress is known to exacerbate the release of pro-inflammatory cytokines (IL-1 $\beta$, tumor necrosis factor- $\alpha$ (TNF- $\alpha$ ) and IL-6), and in this way leads to depressive episodes. In addition, changes of immunologic markers often occur in MDD patients, including increases in pro-inflammatory cytokine activity (e.g. IL-1 $\beta$, TNF- $\alpha$ and IL-6) and inflammation [32,33]. Good antidepressant effects of certain anti-inflammatory therapy are also observed in patients with autoimmune and inflammatory disorders [32]. Thus, the ability of CSP in treating depression is closed related to its anti-inflammatory effects. However, the antiinflammatory mechanism of CSP is unclear. To address this, an Inf-C-T network is established here using all CSP inflammationrelated targets which account for actually more than one-third of all CSP targets and corresponding active compounds, as shown in Figure 6. Based on an initial network pharmacology analysis of this network, it is found that two family proteins, Prostaglandin (PG) synthase and Peroxisome Proliferator-Activated Receptors (PPARs) exert great impacts on inflammation. Therefore, we will explore the anti-inflammatory mechanism of CSP by analyzing the antiinflammatory effects of these two types of targets. PG H synthase, also known as Cyclooxygenase (COX), is the rate-limiting enzyme for synthesis of various endogenous PGs that promote or inhibit acute inflammation. Possessing the highest degree value of 89 in Figure 7 , which depicts the degree distribution of the Inf-C-T network, prostaglandin $\mathrm{H}$ synthase 2 (PTGS2) belongs to the inducible isoform of PG synthase, and has indeed crucial impacts on inflammation. As an important source of PGs synthesis in inflammation, PTGS2 is induced by inflammatory stimuli, hormones, and growth factors. In the presence of pro-inflammatory cytokines, an increased expression of PTGS2 will promote the generation of PG synthase and result in an over PGs synthesis, which may eventually cause depression [34]. In fact, celecoxib, a PTGS2 inhibitor, enhances the efficacy of two antidepressants, fluoxetine and reboxetine, which thus possesses great potential to be a standard antidepressant add-on drug [35]. Besides, chronic administration of celecoxib to the olfactory bulbectomised rat attenuates the increasing trend of pro-inflammatory cytokines in brain [36]. As adjunctive therapy, celecoxib also produces rapid antidepressant effects in patients with bipolar disorder [37]. As shown in Figure 6, in CSP, all those active molecules who interact with PTGS2 are all its inhibitors. In addition, in Inf-C-T network, 39 CSP active components also interact with another PG H synthase, PTGS1 (a constitutively expressed isoform) as inhibitors. In fact, 


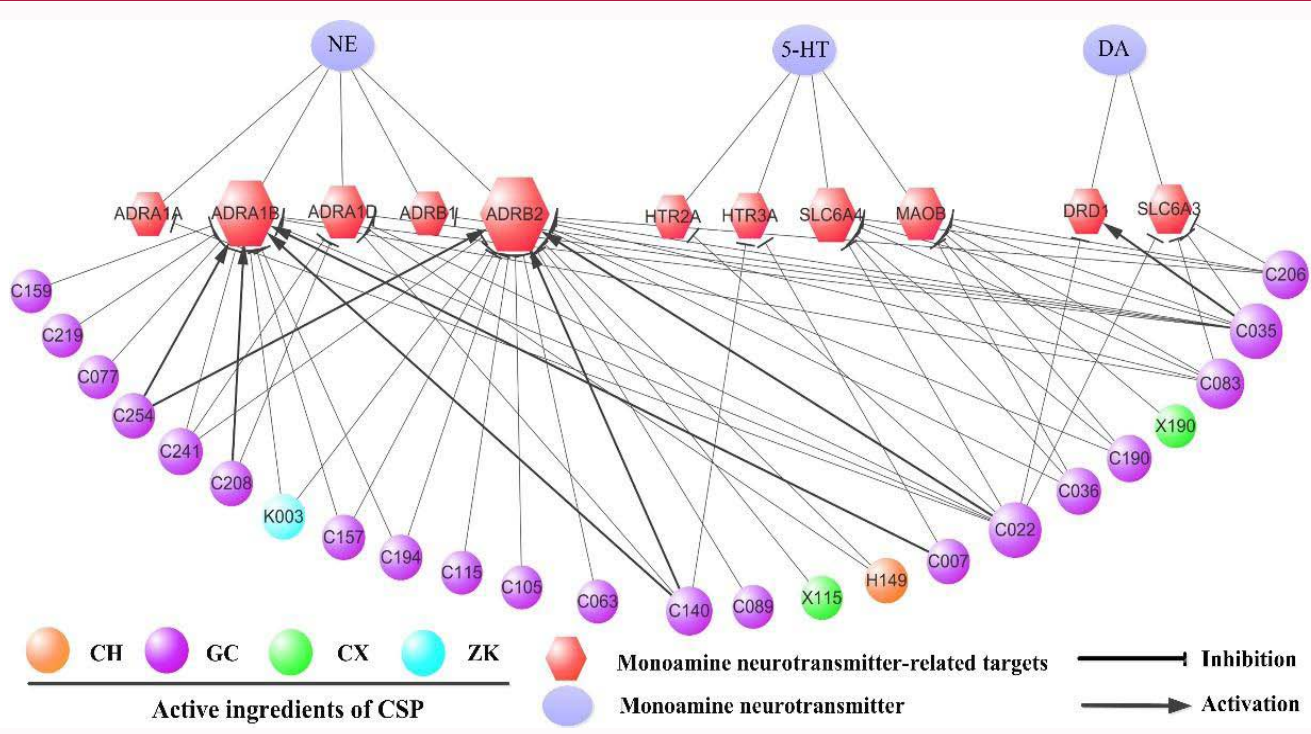

Figure 9: Mon-C-T network of CSP formula, where the ovals, hexagons and circles represent the monoamine neurotransmitters regulated by the targets, the monoamine neurotransmitter-related CSP targets and corresponding active ingredients, respectively. The grey edge represents the interrelationship between the target and the chemical. The node size is proportional to its degree.

PTGS1 is also thought to be induced at sites of inflammation [38]. Some active molecules like glabridin (C157) inhibit both PTGS1 and PTGS2. Therefore, through inhibiting proteins like PGH synthase (PTGS2 and PTGS1) CSP limits the synthesis of PGs, and ultimately inhibits inflammation. In addition, as one of the PPARs subtypes, Peroxisome Proliferator-Activated Receptor Gamma (PPAR $\gamma$, also named $P P A R G$, degree $=77$, Figure $7 \mathrm{~A}$ ) is capable of down regulating the synthesis and release of immunemodulatory cytokines from various cell types that participate in the regulation of inflammatory processes. Thus, PPARG agonists also inhibit the expression of proinflammatory cytokines and exert anti-inflammatory activity in various cell types as shown in Figure 6. All of the 77 active molecules are able to activate $P P A R G$, including luteolin (F094), which has long been reported to reduce IL- 6 production in microglia by inhibiting JNK phosphorylation and activation of AP-1 [39]. Therefore, CSP also exerts a $P P A R G$ agonist-like action to exert an anti-inflammatory action. In conclusion, we believe that the antidepressant effects of CSP lie in to great extent its anti-inflammatory functions through these targets.

Neuroendocrine mechanism: Among CSP targets, six proteins belong to the nuclear receptor subfamily 3, including Glucocorticoid Receptor (GR or NR3C1), the Mineralocorticoid Receptor (MR or NR3C2), Estrogen Receptor Alpha (ESR1), Estrogen Receptor Beta (ESR2), Androgen Receptor (AR) and Progesterone Receptor (PGR). These proteins are all regulatory targets for the HypothalamicPituitary-Adrenal (HPA) axis of human neuroendocrine system. To further explore the influence of CSP on neuroendocrine system, aNeu-C-T network is established using these targets and corresponding active ingredients (Figure 8). First of all, two CSP targets, GR and MR, play a key role in directly regulating the HPA axis function. A short-term activation of HPA axis is normal adaptive responses promoted by stress, while long-term stress makes HPA axis function continuously, which disrupts the negative feedback mechanism of HPA axis and damages the neurological limbic system, ultimately leading to depression. The HPA axis comprises the hypothalamic Paraventricular Nucleus (PVN), the pituitary and the adrenal glands, which is activated during emotional stress.
Under normal circumstances, when human body is stressed, its physiological mechanism is as follows. The hypothalamus secretes Corticotropin Releasing Hormone (CRH) and Arginine Vasopressin (AVP). Then, these two hormones act on the pituitary gland, and increase the secretion of Adrenocorticotropic Hormone (ACTH). ACTH is then delivered to the adrenal cortex in bloodstream and interacts with receptors on adrenocortical cells that stimulate the production and release of cortisol; an adrenal Glucocorticoid stress hormone (also named Glucocorticoid, GC). GC then interacts with GR and MR in HPA tissue, where GR and MR are responsible for feedback inhibition of CRH and ACTH secretion [7]. However, longterm stress could lead to HPA hyper activity and the GC levels in the blood are significantly elevated. Excessive GC continuously binds to GR in the hippocampus, down-regulating glucocorticoid receptors in hippocampal neurons. This results in a destruction of the negative feedback mechanism of the HPA axis, thus failing to effectively inhibit the increased activity of the axis [40]. That is to say, GC keeps HPA in proper activity through MR and GR-mediated feedback modes, and this imbalance in MR/GR mediated effects can lead to depression [40]. Therefore, GR (NR3C1) and MR (NR3C2) are key targets for CSP to regulate neuroendocrine and exert antidepressant effects.

For this reason, GR- and MR- based antidepressants have received more and more attentions from the whole world as they are able to promote the recovery of HPA axis function. Many antidepressants, like Clomipramine (TCA), Fluoxetine (SSRI), Desipramine (TCA), Paroxetine (SSRI), Citalopram (SSRI) and Amitriptyline (TCA), have the function of promoting the GR expression in depressed patients and enhancing GR's ability to bind to GC, and thereby making HPA axis more sensitive to GC feedback and reversing the abnormal behavior of depressed patients [7,41]. GR-based antidepressants can not only repair the HPA axis function, but also shorten the latency of clinical effects as well. For example, only on the fifth day after administration the GR modulator mifepristone produces a significant antidepressant effect [41]. As seen from the Neu-C-T network, GR and MR are also key targets of CSP. Actually, they interact with a total of 11 CSP active ingredients, including H245, H229, H164, H086, S040, S059, X136, C264, C284, F079 and F085. Among them, all those 


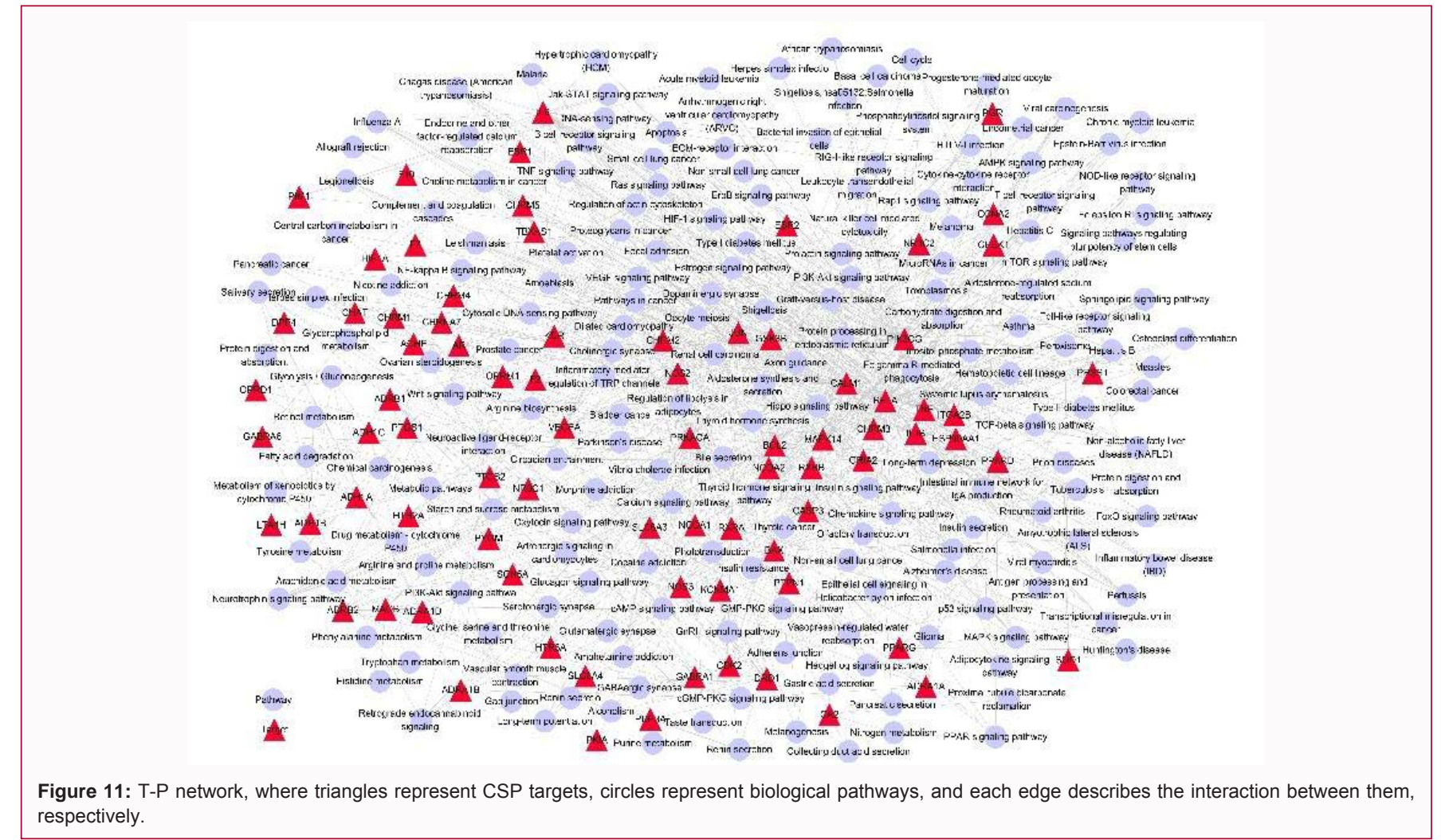

molecules that interact with GR activate this receptor, and thus may exert antidepressant effects similar to mifepristone. Whereas, all those molecules acting on MR have an inhibitory effect. Therefore, these 11 compounds all contribute a lot to CSP's antidepressant efficacy for regulating the function of HPA axis. In fact, Saikosaponin D (H164) has been demonstrated with capability of enhancing the function of HPA axis as well as improving the neurogenesis of hippocampus [42]. Therefore, the antidepressant function of CSP is closely related to the regulation of GR and MR by these active ingredients.

In addition, four gonadotropin receptors (ESR1, ESR2, AR and PGR) are also observed as CSP targets in Neu-C-T network, which are able to alter neuroendocrine responses to stress. Among them, two Estrogen Receptors (ESR1 and ESR2) and one Androgen Receptor (AR) mediate estrogen and androgen, respectively, and in this way affect the activity of adult HPA axis [43]. ESR1 (degree=99, Figure 4B) is one of the most important CSP targets in the treatment of depression. The increased stress sensitivity observed in women is generally due to the decreased sensitivity of estradiol to glucocorticoid-mediated
HPA axis negative feedback. The endogenous estrogen Estradiol (E2) plays a role in inhibiting GC negative feedback, especially through the action of ESR1 on PVN levels [44]. Actually, ESR2 agonists have been shown to attenuate anxiety/despair-like behaviors in rodent models [45]. AR is the second highest target of CSP (degree $=96$, Figure 4B). It mediates androgen, which also has the function of modulating the HPA axis with direct actions on brain regions upstream of PVN to regulate PVN function and neuropeptide expression [43]. As shown in Neu-C-T network, quercetin (H288) is a dual activator of ESR 1 and ESR2 and an inhibitor of AR. In fact, it is capable of inhibiting CRFinduced anxiety and depressive symptoms [46]. Besides, naringenin (P029) is also a dual activator of ESR1 and ESR2 and an inhibitor of $A R$. It has the ability to increase GR, hippocampal 5-HT and NE levels, and reduces serum corticosterone levels in mice exposed to the repeated Tail Suspension Test (TST) [47]. These all demonstrate that CSP exerts antidepressant effects in part by regulating the neuroendocrine system, i.e. by altering the neuroendocrine response to stress via these gonadotropin receptors. 


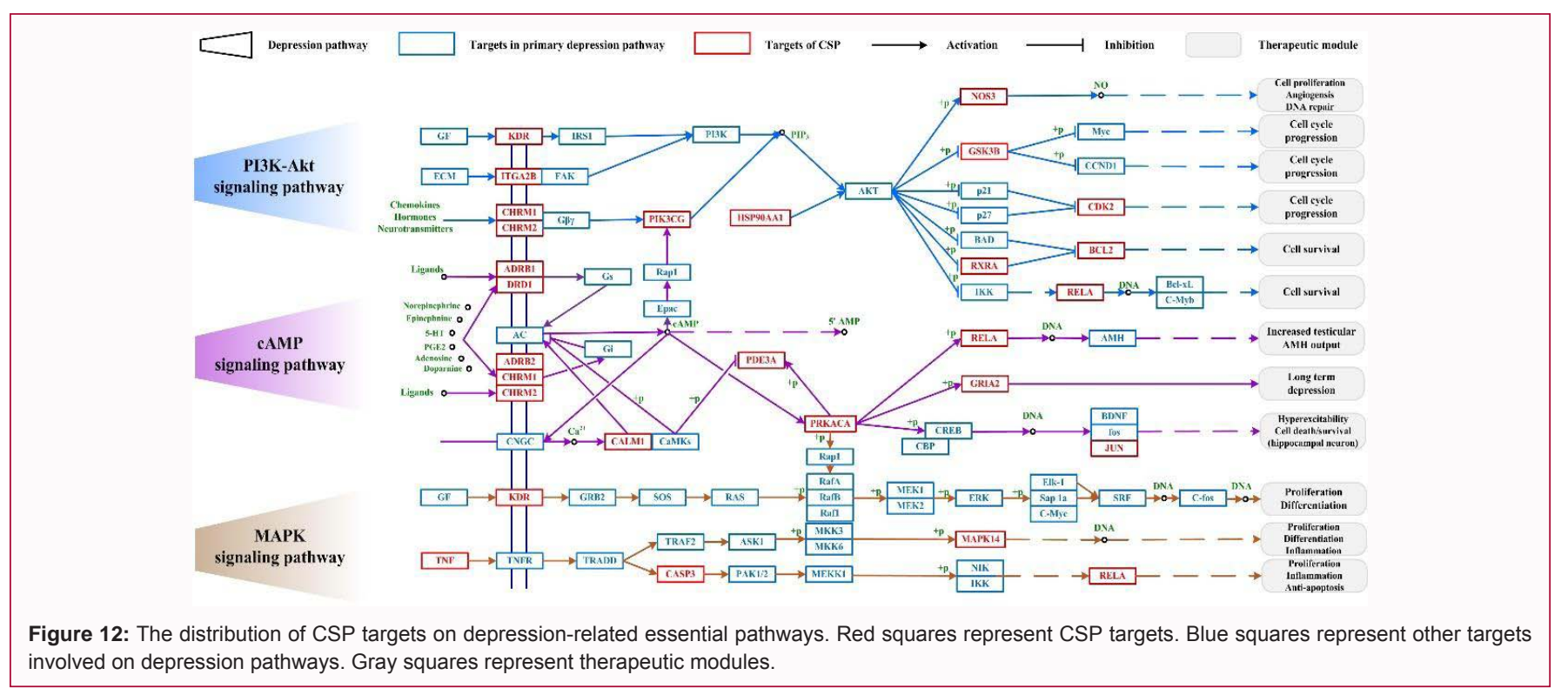

Monoamine neurotransmitter mechanism: Among CSP targets, there are 11 monoamine neurotransmitter receptors, including 5-HT transporter (SLC6A4), 5-HT (HTR2A, HTR3A), Monoamine Oxidase (MAOB), Adrenergic Receptors (ADRA1A, ADRA1B, $A D R A 1 D, A D R B 1$ and $A D R B 2)$, Dopamine (DA) Receptor (DRD1) and Dopamine Transporter (SLC6A3). These receptors are vital for the regulation of monoamine neurotransmitter. Thus, a Mon-C-T network is constructed using all these monoamine neurotransmitterrelated CSP targets with corresponding active compounds to explore the function of CSP in regulating monoamine neurotransmitter in depression treatment (Figure 9). And the degree distribution of all nodes in this network is depicted in Figure 10.

The monoamine neurotransmitter mechanism is the earliest and most widely accepted depression pathogenesis. It is believed that the depression is associated with decreased levels of NE, 5-HT and DA in synaptic space. Therefore, presently, the above 11 targets are divided into NE neurotransmitter receptor, 5-HT neurotransmitter receptor and DA neurotransmitter receptor for analysis and discussion.

NE neurotransmitter receptors include five targets, among which, ADRA1A, ADRA1B and ADRA1D are subtypes of adrenergic a1 receptors ( $\alpha 1-A R s)$, while $A D R B 1$ and $A D R B 2$ are subtypes of adrenergic $\beta$ receptors ( $\beta-A R s)$. On the one hand, activation of $\alpha 1$ $A R$ scan produce an antidepressant effect, since the number of postsynaptic al receptors in rat cortex is significantly increased with long-term administration of antidepressants. Neurogenesis is also enhanced by chronically treating wild type mice with the $\alpha 1 \mathrm{~A}-A R$ agonist, cirazoline $[48,49]$. On the other hand, the down-regulation of $\beta-A R$ s is also a marker of anti-depression in depression patients [48]. NE Reuptake Inhibitors (NRIs) clinically increase synaptic NE concentrations by inhibiting NE from reuptake in the synaptic cleft, playing an important role in the treatment of depression. The MonC-T network analysis indicates that a total of 25 molecules interact with NE targets, such as K003, H149, C022, C035, etc. Among them, 4 molecules activate a1-ARs. Besides, 17 molecules inhibit $\beta$-ARs, such as X115 and C063, who inhibit ADRB2 with similar roles as NE inhibitors. Therefore, it is inferred that the antidepressant effect of CSP is also attributed to the regulation of NE receptors by CSP active ingredients.
5-HT neurotransmitter receptors involve SLC6A4, HTR2A, HTR3A and MAOB, among which SLC6A4 is a 5-HT transporter (Serotonin Transporter, SERT), HTR2A (5-HT2A) and HTR3A (5-HT2C) are both subtypes of 5-HT2 receptor and $M A O B$ is a monoamine oxidase. As a matter of fact, SLC6A4 receptor has long been recognized as an important target for depression treatment that $80 \%$ antidepressants in the market are SSRIs based antidepressants, whichinhibit5-HT transporters from the reuptake of 5-HT in the synaptic cleft and increase 5-HT concentration [28]. It can be seen from that C083, C190, C022, C206, C035 and C036 indirectly affect the 5-HT neurotransmitter through inhibiting SLC6A4, and may thereby exert antidepressant effects (Figure 10). Secondly, the fact that a great deal of antidepressant drugs, e.g., TCA, SSRI, mirtazapine and mianserin, reduce the receptor density of HTR $2 A$ in the forebrain and improve the symptoms of depression, also indicates that the HTR2A receptor may be a potential anti-depression target [29]. Actually, the pathological enhancement of HTR2A receptor's function in the limbic system (upregulation of hypersensitivity) causes a reduction in the release of 5-HT synthesis, which leads to depressive symptoms. Therefore, C022 which can inhibit HTR2A deserves more attentions of research. In addition, depressive symptoms also appear when HTR3A receptor hypersensitivity is upregulated. HTR $3 A$ receptor antagonists can not only increase 5-HT levels, but also indirectly increase DA and NE levels, improving depressive symptoms due to monoamine neurotransmitter reduction as shown in Figure 10 [50,51]. C007 not only inhibits HTR $3 A$ but also activates $A D R A 1 B$, so C007 may exert a good antidepressant effect. As for $M A O B$, it is actually the target of the antidepressant MAOIs. The latter exerts antidepressant effects by inhibiting isoenzymes (MAO-A and MAO-B) to hinder 5 -HT reuptake and inhibit 5 -HT degradation. Thus, the six active compounds that inhibit MAOB, including C083, C190, C206, C035, C036 and X190, have all potentials to be antidepressants in Figure 10.

The third class of DA neurotransmitter receptors involved in CSP includes DRD1, a D1 receptor and SLC6A3, a dopamine transporter. The main role of DRD1 is to increase the release of DAtype neurotransmitter from cells, and thereby achieve antidepressant effects. SLC6A3 is also a susceptibility gene for depression, which mediates the reuptake of dopamine from the synaptic cleft and is a principal regulator of dopaminergic neurotransmission. The new 


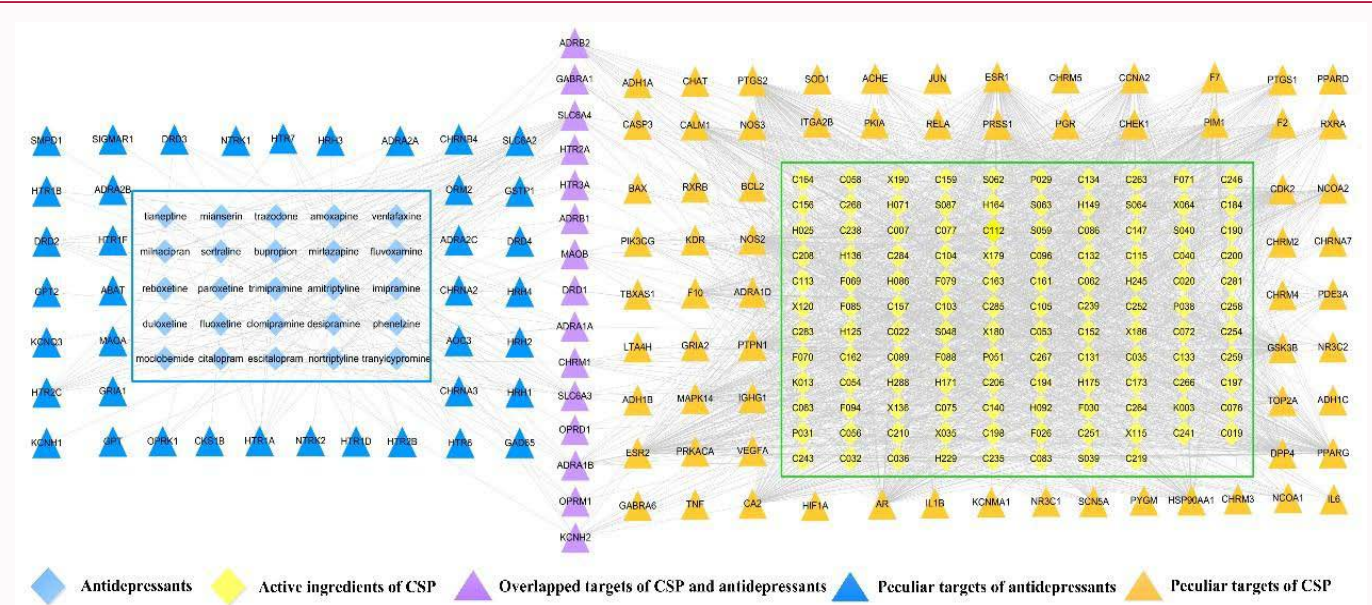

Figure 13: D-T network, which is composed of all the antidepressants (blue diamonds) and corresponding targets (blue triangles), all CSP active ingredients (yellow diamonds) and corresponding targets (yellow triangles) and the overlapped targets of CSP and antidepressants (purple triangle nodes).

antidepressant bupropion exerts antidepressant effects mainly through reducing the reuptake of DA and increasing the level of DA [51]. Actually, all antidepressants, to some extent, all affect dopamine in the frontal area of the brain and in the nucleus accumbens as shown in Figure 10 [30]. DRD1 is activated by C035, whereas SLC6A3 is inhibited by C083, C022, C206 and C035 compounds. Thus, all of them may mediate DA neurotransmitter levels to exert antidepressant effects.

It is worth noting that some molecules inhibit multiple targets to regulate a variety of neurotransmitters, and thereby may exert a strengthened antidepressant effect. For example, C190 simultaneously suppresses MAOB, SLC6A4, and ADRB2. C036 is capable of inhibiting $M A O B, S L C 6 A 4$, and $A D R B 2$ at the same time. As a result, they may increase the concentration of NE and 5-HT simultaneously and thus deserve more in-depth research. In summary, the synergistic effect of CSP in the treatment of depression attribute, in essence, to its capability of anti-inflammation and the regulation effects of neuroendocrine and monoamine neurotransmitter systems.

\section{Uncovering the synergy from pathway level}

Drugs not only act on targets, but also in this way affect various pathways that these targets are involved in associated with specific diseases. In addition to the diversity of herbal active compounds and targets, biological pathways are also crucial for analyzing the complex mechanisms of herbal therapy. Therefore, to investigate the antidepressant the therapeutic mechanism of CSP from pathway level, a Target-Pathway (T-P) network is established, using 192 biological pathways that are obtained by pathway enrichment analysis employing all CSP targets, as shown in Figure 11. In this network, three pathways, i.e., the PI3K-Akt, cAMP and MAPK signaling pathways have attracted our attention. Because they are not only closely related to CSP targets (i.e. with high degree $=13,12,7$ ), but also closely associated with the pathogenesis of depression. Therefore, they are integrated for further analysis (Figure 12).

PI3K-Akt signaling pathway: It is known that PI3K/Akt signaling pathway inhibits inflammation and anti-inflammation is an indispensable strategy for depression treatment as shown in Figure 12. The 12 CSP targets are all important regulators of inflammatory response that are involved in PI3K-Akt signaling pathway. Among them, some proteins are key targets for upstream and downstream of the pathway, such as upstream target PIK3CG and downstream InfC-T network as shown in the Figure 6. Both PIK3CG and GSK3B are significant targets for anti-inflammatory effects, interacting with 14 and 81 CSP active compounds, respectively. Upstream target PIK3CG has the ability in upregulating anti-inflammatory cytokines and inhibiting the expression of pro-inflammatory cytokines [52]. And the downstream target GSK3B is also a vital target for the regulation of inflammatory cell proliferation. Its inhibitors can effectively reduce pro-inflammatory cytokines and increase anti-inflammatory cytokines. In fact, among the numerous CSP molecules that interact with these 12 CSP targets, glycyrrhizic acid (C283) has been proven reducing inflammation through the PI3K-Akt-GSK3B pathway [53]. These indicate that the PI3K-Akt signaling pathway contributes a lot for CSP to exert anti-inflammatory effects.

cAMP signaling pathway: CAMP signaling pathway plays a vital role in depression treatment as it mediates monoamine responses. Actually, a number of neurotransmitter receptors, including serotonergic, adrenergic and dopamine, utilize this pathway to function efficiently. 11 CSP targets are observed being involved in this pathway, including several keyup streams (like DRD1 and ADRB1/2) and downstream (like $P R K A C A$ ) monoamine regulators from Figure 12. As shown in the Mon-C-T network, DRD1 and ADRB1/2, as the upstream membrane proteins of cAMP signaling pathway, are vital receptors of monoamine neurotransmitters for CSP to exert antidepressant effects through the monoamine neurotransmitter mechanism. Actually, one major function of $D R D 1$ and $A D R B 1 / 2$ is to increase cAMP levels, thereby activating downstream PRKACA. Subsequently, PRKACA phosphorylates cytoplasm and nuclear proteins, regulates cellular metabolism, increases the release of DA and NE neurotransmitter, and ultimately achieves antidepressant effects [7]. Isoflavone (C035) activates DRD1 and inhibits $A D R B 1$, while Cubebin (H149) and Myricanone (X115) inhibit ADRB2, and in this way the concentrations of monoamine neurotransmitters DA and NE may both be increased as seen from Figure 9. In fact, typical antidepressants also activate the cAMP signal transduction pathway through various monoamine neurotransmitter receptors [54]. Thus, the enrichment of CSP's targets on cAMP signaling pathway also indicates that similar to most antidepressants, the regulation of monoamine neurotransmitter concentrations via this signaling pathway is also a critical path for CSP to produce antidepressant effects. 


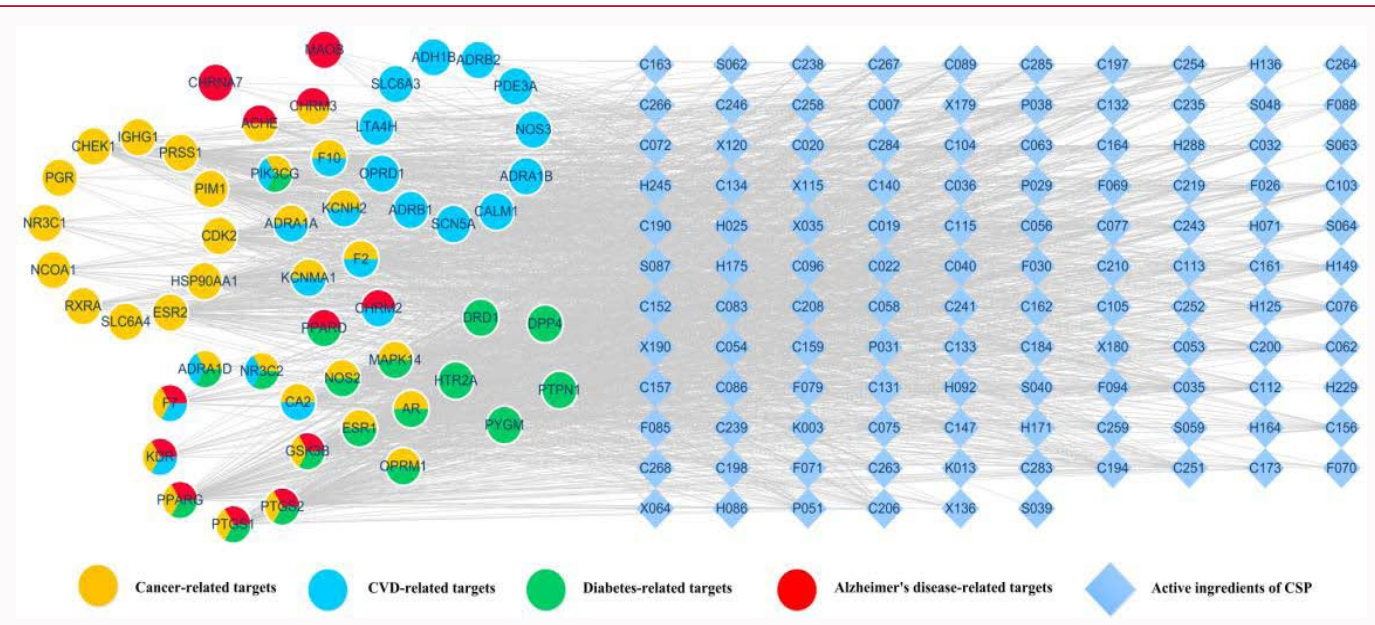

Figure 14: C-T-D network of CSP in the treatment of complications of depression, where the circles and diamonds represent complication-associated targets and corresponding active ingredients, respectively. The grey edge represents the interrelationship between the target and the chemical.

Table 2: Adverse reactions of all current antidepressants.

\begin{tabular}{|c|c|c|c|c|}
\hline САТ & Drug & Primary receptor & Pharmacological action & Adverse reactions \\
\hline \multirow{6}{*}{1} & Fluoxetine & \multirow{6}{*}{$5-\mathrm{HT}$} & \multirow{6}{*}{ Selective 5-HT reuptake inhibitors (SSRIs) } & \multirow{6}{*}{$\begin{array}{l}\text { Nausea, vomiting, gastrointestinal bleeding, headache, dry mouth, } \\
\text { insomnia, agitation, sweating, dizziness and sexual dysfunction. }\end{array}$} \\
\hline & Paroxetine & & & \\
\hline & Fluvoxamine & & & \\
\hline & Citalopram & & & \\
\hline & Sertraline & & & \\
\hline & Escitalopram & & & \\
\hline 2 & Trazodone & $5-\mathrm{HT}$ & $\begin{array}{l}5-\mathrm{HT} 2 \mathrm{~A} / \mathrm{C} \text { receptor antagonists and } 5-\mathrm{HT} \\
\text { reuptake inhibitors (SARIs) }\end{array}$ & $\begin{array}{l}\text { Dizziness, headache, nausea, dry mouth, constipation or diarrhea, } \\
\text { insomnia, rash, etc. }\end{array}$ \\
\hline 3 & Tianeptine & $5-\mathrm{HT}$ & $5-\mathrm{HT}$ reuptake agonist (SSRA) & $\begin{array}{l}\text { Dry mouth, constipation, insomnia, dreams, dizziness, weight gain, } \\
\text { nervousness, nausea, etc. }\end{array}$ \\
\hline 4 & Reboxetine & NE & NE reuptake inhibitors (NaRIs) & $\begin{array}{l}\text { Nausea, dry mouth, insomnia, sweating, constipation, insomnia, } \\
\text { headache, etc. }\end{array}$ \\
\hline \multirow{3}{*}{5} & Amoxapine & \multirow{3}{*}{ NE } & \multirow{3}{*}{ TCAs as NE reuptake inhibitors } & \multirow{3}{*}{ Anti muscarinic and cardiac adverse reactions. } \\
\hline & Desipramine & & & \\
\hline & Nortriptyline & & & \\
\hline \multirow{4}{*}{6} & Amitriptyline & \multirow{4}{*}{ 5-HT, NE } & \multirow{4}{*}{ TCAs as NE and $5-\mathrm{HT}$ reuptake inhibitors } & \multirow{4}{*}{$\begin{array}{l}\text { Anti cholinergic, central nervous system and cardiovascular side } \\
\text { effects, sexual side effects, weight gain, allergic reactions and } \\
\text { poisoning. }\end{array}$} \\
\hline & Clomipramine & & & \\
\hline & Imipramine & & & \\
\hline & Trimipramine & & & \\
\hline 7 & Mirtazapine & 5-HT, NE & $\begin{array}{l}\text { NE and specific 5-HT reuptake inhibitors } \\
\text { (NaSSAs) }\end{array}$ & Increased appetite, weight gain, lethargy, excessive sedation. \\
\hline 8 & Mianserin & 5-HT, NE & $\begin{array}{l}\text { blocking a adrenergic receptor, serotonin } \\
\text { receptor (HCA) }\end{array}$ & $\begin{array}{l}\text { Anti muscarinic and cardiac adverse reactions, dizziness, weak, rare } \\
\text { neutropenia. }\end{array}$ \\
\hline \multirow{3}{*}{9} & Venlafaxine & \multirow{3}{*}{ 5-HT, NE } & \multirow{3}{*}{ 5-HT and NE reuptake inhibitors (SNRIs) } & \multirow{3}{*}{$\begin{array}{l}\text { Nausea, lethargy, sweating, dizziness, sexual dysfunction, } \\
\text { hypertension, anxiety, dry mouth, dizziness, constipation, sexual } \\
\text { syndrome and serotonin syndrome etc. }\end{array}$} \\
\hline & Duloxetine & & & \\
\hline & Milnacipran & & & \\
\hline 10 & Bupropion & NE, DA & $\begin{array}{l}\text { NE and DA reuptake and receptor inhibitors } \\
\text { (NDRIs) }\end{array}$ & $\begin{array}{l}\text { Neuropsychiatric disorders, agitation, mental disorders, } \\
\text { gastrointestinal dysfunction, nausea, vomiting, nervous system } \\
\text { dysfunction. }\end{array}$ \\
\hline \multirow{3}{*}{11} & Iproniazid & \multirow{3}{*}{ MAOA, MAOB } & \multirow{3}{*}{$\begin{array}{l}\text { Irreversible monoamine oxidase inhibitors } \\
\text { (MAOls) }\end{array}$} & \multirow{3}{*}{ Causing hypertensive crisis. } \\
\hline & Tranylcypromine & & & \\
\hline & Phenelzine & & & \\
\hline 12 & Moclobemide & MAOA, MAOB & $\begin{array}{l}\text { Reversible monoamine oxidase inhibitor } \\
\text { (RIMA) }\end{array}$ & Headache, dizziness, nausea. \\
\hline
\end{tabular}

MAPK signaling pathway: MAPK signaling pathway plays a crucial role in many aspects of immune mediated inflammatory responses [55]. As shown in Figure 12, 5 CSP targets are involved in the regulation of MAPK signaling pathway. Among them, MAPK14 is one of the indispensable targets and is considered to be a central regulator of an inflammatory response in multiple cell types [56]. As shown in the Inf-C-T network, a total of 66 CSP active ingredients like isotrifoliol (C062), quercetin (H288) and kaempferol (S064) 
inhibit MAPK14. Actually, isotrifoliol significantly suppresses the expression of inflammatory mediators in Lipopolysaccharide (LPS)induced RAW 264. 7 macrophages through MAPK signaling pathway and exerts anti-inflammatory effect [42]. Besides, MAPK14 can also be activated by IL- $1 \beta$ and TNF- $\alpha$ and in this way significantly increase the reuptake of 5-HT (as demonstrated in rat brain synaptosomes [32]) and decrease synaptic availability of 5-HT, which ultimately leads to the depression-like behavior in experimental animals. These results manifest that CSP presents a significant effect on gene expression of inflammatory mediators, as well as the activity of 5-HT membrane transporters by regulating the MAPK signaling pathway.

The concept of pathway cross-talk refers to protein interactions shared between distinct pathways [57]. As we can see from Figure 12 , approximately $23 \%(6 / 26)$ of CSP targets participate in two or more pathways simultaneously. Among them, PIK3CG is contained in both cAMP and PI3K-Akt signaling pathways, which is required for chemokine-dependent migration of neutrophils, macrophages and mast cells to eliminate infection [58]. Actually, 14 CSP active molecules, such as S064, F026, F071, X120, P031, etc., are capable of modulating PIK3CG. Among them, some molecules such as liquiritin (C152) have both anti-inflammatory and monoamine neurotransmitter regulation functions. In fact, C152 not only has strong inflammatory effects [59], but also significantly increases $\mathrm{NE}$ and 5-HT levels and lowers the ratio of 5-HIAA/5-HT in the hypothalamus, hippocampus and cortex $[6,60]$. These facts indicate that it may be due to the presence of cross-talk proteins that enables CSP molecules to regulate multiple pathways to play synergistic roles.

Taken together, these findings reveal that antidepressant functions of CSP are achieved just through the complex pathway network and especially those cross-talk targets.

\section{CSP synergistic actions on side effect and complication}

Side effect: In general, there are currently more than a dozen types of antidepressants available on the market. However, although they have antidepressant activity, they still possess some side effects, as shown in Table 2. Here, to better explore the CSP synergy in depression treatment, a Drug-Target (D-T) network is constructed, as shown in Figure 13. As shown in Table 2, common side effects, e.g., nausea, diarrhea, dyspepsia, gastrointestinal bleeding and abdominal pain, are often observed after taking antidepressants such as SSRIs and SNRIs, which are mainly caused by the major regulatory role of serotonin in the motor and sensation of the gastrointestinal tract [6]. In fact, two serotonin relevant proteins (HTR2A, HTR3A) are found to be common targets for antidepressants and CSP (Figure 13). Since CSP active compounds, such as quercetin (C022) and in ermine (C007) inhibit HTR2A and HTR3A targets respectively in C-T network, nausea and vomiting should also be observed theoretically when taking CSP. However, there have been no reports about CSP's side effects so far, which may be due to the fact that many CSP active compounds also act on the targets of gastrointestinal diseases and thus restrict or offset the side effects of gastrointestinal diseases. In fact, 16 CSP targets are closely associated with gastrointestinal diseases. Among them, AR plays an important role in gastric cancers for it is involved in tumor progression. It also promotes the esophageal cancer cell migration and proliferation via Matrix metalloproteinase. From Figure 3 we can find that a total of 96 CSP active molecules such as kaempferol (S064) and sugeonylacetate (F088) interact with AR. Besides, as seen from the Neu-C-T network, S064 and F088 are identified to block $\mathrm{AR}$, highlighting therapeutic effects on the stomach diseases. Therefore, some of the molecules in CSP play important roles in regulating these gastrointestinal related targets, thereby reducing or even eliminating related side effects. In fact, CSP is widely used clinically in the treatment of gastrointestinal diseases [61].

Complication: Depressive disease has a high mortality rate, which is due to not only the suicidal behavior in patients with depression, but also the various complications. There are four main types of complications common to depression, including cardiovascular disease, Alzheimer's disease, diabetes and cancer. In fact, according to the human disease network, different diseases have a common genetic origin to some extent [7]. This is also the reason for the recurrent episodes of depression when only antidepressants are used to treat depression. CSP is different from current antidepressants in the treatment of depression, since it is also capable of treating certain depression complications. To address this, we established a Compound-Target-Disease (C-T-D) network by mapping all CSP targets to their associated diseases and active ingredients (Figure 14). It shows that up to $65 \%(54 / 83)$ targets are associated with these four major depression complications, among which cancer (34) ranks first and CVD (24) the second Figure 14. Since cancer has the most targets in Figure 14, we will take it as an example for illustration. In fact, CSP has a long history of clinical use in the treatment of cancers $[62,63]$, which may be due to some key targets such as PTGS1/2 and ER. PTGS1 and PTGS2 play important roles in carcinogenesis and angiogenesis in human tumors. Epidemiological studies and early clinical trials have also shown that administration of dual PTGS1/PTGS2 or selective PTGS2 inhibitors reduces the risk of cancer development. As shown in Inf-C-T network, on the one hand, PTGS2 interacts with up to 89 active ingredients, all of which are inhibitors. On the other hand, some of these PTGS2 inhibitors are also inhibitors of PTGS1, i.e. the dual inhibitors. Thus, they may endow CSP anticancer function. Similar to cancers, it is the diversity of CSP active ingredients acting on the disease-related targets that play a synergistic therapeutic role in the treatment of depression complications.

In summary, comparing to current antidepressants, CSP can not only treat or even avoid some side effects (e.g. gastrointestinal system diseases), but also be capable of treating certain complications such as cardiovascular diseases and cancers. This provides a new idea for the extraction of effective active ingredients from CSP for drug combination development and treatment of depression.

\section{Conclusion}

In this paper, a SP approach is employed to study the CSP's synergic mechanisms in depression treatment. The characteristics of complementary and synergic actions of CSP active compounds for depression treatment are investigated from the perspectives of "multi-compound", "multi-target" and "multi-mechanism", as well as the signaling pathway, side effect and complication. The research conclusions are as follows:

1. Screened from 1,095 components in 11 herbs, a total number of 126 active ingredients are identified as candidate compounds of CSP formula, and 83 proteins are identified as CSP targets. This indicates the "multi-compound" and "multiple targets" features of CSP prescription.

2. We established a PPI network, and the analysis results show that CSP antidepressant efficacy is partly attributed to its direct interaction with its over-lapped targets with antidepressants, and 
partly depends on greatly the direct or indirect interactions of its active ingredients with clinical antidepressants' targets.

3. Through the construction and analysis of C-T network, it is found that the synergistic effects of CSP in the treatment of depression are attributed to its three mechanisms, i.e., the antiinflammation as well as the regulation of neuroendocrine system and of monoamine neurotransmitter. Moreover, the implementation of these mechanisms relies on the smooth operation of complex biological pathway networks, including especially PI3K-Akt, cAMP and MAPK signaling pathways.

4. The analysis of D-T and C-T networks reveals that CSP is also capable of relieving or even certain eliminating side effects and complications of depression. The reason is that the active ingredients also act on targets of a variety of related diseases such as gastrointestinal diseases, cancer, etc.

In conclusion, these findings may be of help for identifying novel compounds and targets for antidepressants design and promote the development of antidepressant treatments in a more efficient way.

\section{Author Contributions}

Yujing Zhao established the model, implemented the methods and analyzed the results, and wrote this paper. Qilei Liu revised the English grammar of the paper. Feng Li and Yan Li provided ideas and text corrections for the paper. The final draft read and approved by all authors.

\section{Acknowledgement}

This work was supported by the National Natural Science Foundation of China [81530100].

\section{References}

1. Haase J, Brown E. Integrating the monoamine, neurotrophin and cytokine hypotheses of depression-a central role for the serotonin transporter? Pharmacol Ther. 2015;147:1-11.

2. Dean J, Keshavan M. The neurobiology of depression: An integrated view. Asian J Psychiatr. 2017;27:101-11.

3. Carvalho AF, Sharma MS, Brunoni AR, Vieta E, Fava GA. The safety, tolerability and risks associated with the use of newer generation antidepressant drugs: a critical review of the literature. Psychother Psychosom. 2016;85(5):270-88.

4. Haenisch B, Bonisch H. Depression and antidepressants: insights from knockout of dopamine, serotonin or noradrenaline re-uptake transporters. Pharmacol Ther. 2011;129(3):352-68.

5. Penn E, Tracy DK. The drugs don't work? Antidepressants and the current and future pharmacological management of depression. Ther Adv Psychopharmacol. 2012;2(5):179-88.

6. Liu H. Investigation on the antidepression effect of Compound Chaigui Fang. Shanxi University. 2016.

7. Grosso C, editor. Herbal medicine in depression: traditional medicine to innovative drug delivery. Springer; 2016:1-16.

8. Huang YS, Luo RH, Yuan ZX. Clinical study of chaihu shugan powder in the treatment of depression after apoplexy. J Practical Traditional Chinese Internal Med. 2012;7:21.

9. Wang Y, Fan R, Huang X. Meta-analysis of the clinical effectiveness of traditional Chinese medicine formula Chaihu-Shugan-San in depression. J Ethnopharmacol. 2012;141(2):571-7.

10. Yeung WF, Chung KF, Ng KY, Yu YM, Ziea ET, Ng BF. A systematic review on the efficacy, safety and types of Chinese herbal medicine for depression. J Psychiatr Res. 2014;57:165-75.

11. Sun Y, Xu X, Zhang J, Chen Y. Treatment of depression with Chai $\mathrm{Hu}$ Shu Gan San: a systematic review and meta-analysis of 42 randomized controlled trials. BMC Complement Altern Med. 2018;18(1):66.

12. Zhang K, Pan X, Wang F, Ma J, Su G, Dong Y, et al. Baicalin promotes hippocampal neurogenesis via SGK1-and FKBP5-mediated glucocorticoid receptor phosphorylation in a neuroendocrine mouse model of anxiety/ depression. Sci Rep. 2016;6:30951.

13. Zheng C, Pei T, Huang C, Chen X, Bai Y, Xue J, et al. A novel systems pharmacology platform to dissect action mechanisms of traditional Chinese medicines for bovine viral diarrhea disease. Eur J Pharm Sci. 2016;94:33-45.

14. Li P, Chen J, Wang J, Zhou W, Wang X, Li B, et al. Systems pharmacology strategies for drug discovery and combination with applications to cardiovascular diseases. J Ethnopharmacol. 2014;151(1):93-107.

15. Ru J, Li P, Wang J, Zhou W, Li B, Huang C, et al. TCMSP: a database of systems pharmacology for drug discovery from herbal medicines. J Cheminform. 2014;6(1):13

16. Veber DF, Johnson SR, Cheng HY, Smith BR, Ward KW, Kopple KD. Molecular properties that influence the oral bioavailability of drug candidates. J Med Chem. 2002;45(12):2615-23.

17. Madden J C. In silico approaches for predicting ADME properties. in recent advances in QSAR studies. Springer. 2010;8:283-304.

18. Clark DE, Pickett SD. Computational methods for the prediction of 'druglikeness'. Drug Discov Today. 2000;5(2):49-58.

19. Guney E, Menche J, Vidal M, Barábasi AL. Network-based in silico drug efficacy screening. Nat Commun. 2016;7:10331.

20. Dennis G, Sherman BT, Hosack DA, Yang J, Gao W, Lane HC, et al. DAVID: database for annotation, visualization, and integrated discovery. Genome Biol. 2003;4(5):P3.

21. Merico D, Isserlin R, Stueker O, Emili A, Bader GD. Enrichment map: A network-based method for gene-set enrichment visualization and interpretation. PLoS One. 2010;5(11):e13984.

22. Kanehisa M, Furumichi M, Tanabe M, Sato Y, Morishima K. KEGG: New perspectives on genomes, pathways, diseases and drugs. Nucleic Acids Res. 2017;45(D1):D353-61.

23. Liu H, Wang J, Zhou W, Wang Y, Yang L. Systems approaches and polypharmacology for drug discovery from herbal medicines: an example using licorice. J Ethnopharmacol. 2013;146(3):773-93.

24. Thiyagarajan P, Chandrasekaran CV, Deepak HB, Agarwal A. Modulation of lipopolysaccharide-induced pro-inflammatory mediators by an extract of Glycyrrhiza glabra and its phytoconstituents. Inflammopharmacology. 2011;19(4):235-41.

25. Hosseinzadeh H, Nassiri-Asl M. Pharmacological effects of Glycyrrhiza spp. and its bioactive constituents: update and review. Phytother Res. 2015;29(12):1868-86.

26. Wang W, Hu X, Zhao Z, Liu P, Hu Y, Zhou J, et al. Antidepressantlike effects of liquiritin and isoliquiritin from Glycyrrhiza uralensis in the forced swimming test and tail suspension test in mice. Prog Neuropsychopharmacol Biol Psychiatry. 2008;32(5):1179-84.

27. Gong S, Zhang J, Guo Z, Fu W. Senkyunolide A Protects CorticosteroneInduced Cell Apoptosis Via Modulating Protein Phosphatase 2A and a-Synuclein. BioRxiv. 2017;1:145326.

28. Celada P, Puig MV, Amargós-Bosch M, Adell A, Artigas F. The therapeutic role of 5-HT1A and 5-HT2A receptors in depression. J Psychiatry Neurosci. 2004;29(4):252-65.

29. Berg KA, Harvey JA, Spampinato U, Clarke WP. Physiological and 
therapeutic relevance of constitutive activity of 5-HT2A and 5-HT2C receptors for the treatment of depression. Prog Brain Res. 2008;172:287305 .

30. Lopez-Leon S, Janssens ACJW, Ladd AGZ, Del-Favero J, Claes SJ, Oostra $\mathrm{BA}$, et al. Meta-analyses of genetic studies on major depressive disorder. Mol Psychiatry. 2008;13(8):772-85.

31. Azuaje FJ, Zhang L, Devaux Y, Wagner DR. Drug-target network in myocardial infarction reveals multiple side effects of unrelated drugs. Sci Rep. 2011;1:52.

32. Miller AH, Maletic V, Raison CL. Inflammation and its discontents: the role of cytokines in the pathophysiology of major depression. Biol Psychiatry. 2009;65(9):732-41.

33. Hurley LL, Tizabi Y. Neuroinflammation, neurodegeneration, and depression. Neurotox Res. 2013;23(2):131-44.

34. Ricciotti E, FitzGerald GA. Prostaglandins and inflammation. Arterioscler Thromb Vasc Biol. 2011;31(5):986-1000.

35. Feng DW. Progress of development and research on targets and drugs for major depressive disorder. Chinese Hospital Pharmaceutical J. 2018;38(4):443-9.

36. Myint AM, Steinbusch HW, Goeghegan L, Luchtman D, Kim YK, Leonard BE. Effect of the COX-2 inhibitor celecoxib on behavioural and immune changes in an olfactory bulbectomised rat model of depression. Neuroimmunomodulation. 2007;14(2):65-71.

37. Zhou NN, Dai R, Lin Q. New target for treatment of depression: COX-2. J Psychiatry. 2009;22(02):153-7.

38. Bertolini A, Ottani A, Sandrini M. Dual acting anti-inflammatory drugs: a reappraisal. Pharmacol Res. 2001;44(6):437-50.

39. Jang S, Kelley KW, Johnson RW. Luteolin reduces IL-6 production in microglia by inhibiting JNK phosphorylation and activation of AP-1. Proc Natl Acad Sci U S A. 2008;105(21):7534-9.

40. Zhao H, Yang DS. Regulation of central stress response by corticosteroid receptor system. Foreign Medicine. Psychiatry. 2003;30(1):55-9.

41. Liu JL, Wan YH, Chen NH. Research Progress in the treatment of depression. Chinese Pharmacological Bulletin. 2011;27(09):1193-6.

42. Li HY, Zhao YH, Zeng MJ, Fang F, Li M, Qin TT, et al. Saikosaponin $\mathrm{D}$ relieves unpredictable chronic mild stress induced depressive-like behavior in rats: Involvement of HPA axis and hippocampal neurogenesis. Psychopharmacology (Berl). 2017;234(22):3385-94.

43. Heck AL, Handa RJ. Sex differences in the hypothalamic-pituitaryadrenal axis' response to stress: an important role for gonadal hormones. Neuropsychopharmacology. 2019;44(1):45-58.

44. Weiser MJ, Handa RJ. Estrogen impairs glucocorticoid dependent negative feedback on the hypothalamic-pituitary-adrenal axis via estrogen receptor alpha within the hypothalamus. Neuroscience. 2009;159(2):883-95.

45. Donner N, Handa RJ. Estrogen receptor beta regulates the expression of tryptophan-hydroxylase 2 mRNA within serotonergic neurons of the rat dorsal raphe nuclei. Neuroscience. 2009;163(2):705-18.

46. Bhutada P, Mundhada Y, Bansod K, Ubgade A, Quazi M, Umathe S, et al Reversal by quercetin of corticotrophin releasing factor induced anxietyand depression-like effect in mice. Prog Neuropsychopharmacol Biol Psychiatry. 2010;34(6):955-60.

47. Yi LT, Li J, Li HC, Su DX, Quan XB, He XC, et al. Antidepressant-like behavioral, neurochemical and neuroendocrine effects of naringenin in the mouse repeated tail suspension test. Prog Neuropsychopharmacol Biol Psychiatry. 2012;39(1):175-81.
48. Elhwuegi AS. Central monoamines and their role in major depression. Prog Neuropsychopharmacol Biol Psychiatry. 2004;28(3):435-51.

49. Doze VA, Handel EM, Jensen KA, Darsie B, Luger EJ, Haselton JR, et al. a1A-and $\alpha 1 B$-adrenergic receptors differentially modulate antidepressantlike behavior in the mouse. Brain Res. 2009;1285:148-57.

50. Yang W, Wang Q, Kanes SJ, Murray JM, Nishikura K. Altered RNA editing of serotonin 5-HT2C receptor induced by interferon: implications for depression associated with cytokine therapy. Brain Res Mol Brain Res. 2004;124(1):70-8.

51. Millan MJ. Serotonin 5-HT2C receptors as a target for the treatment of depressive and anxious states: focus on novel therapeutic strategies. Therapie. 2005;60(5):441-60.

52. Kitagishi Y, Kobayashi M, Kikuta K, Matsuda S. Roles of PI3K/AKT/ GSK3/mTOR pathway in cell signaling of mental illnesses. Depress Res Treat. 2012;2012:752563.

53. Kao TC, Shyu MH, Yen GC. Glycyrrhizic acid and $18 \beta$-glycyrrhetinic acid inhibit inflammation via PI3K/Akt/GSK3 $\beta$ signaling and glucocorticoid receptor activation. J Agric Food Chem. 2010;58(15):8623-9.

54. Conti AC, Cryan JF, Dalvi A, Lucki I, Blendy JA. cAMP response element-binding protein is essential for the upregulation of brain-derived neurotrophic factor transcription, but not the behavioral or endocrine responses to antidepressant drugs. J Neurosci. 2002;22(8):3262-8.

55. Hommes DW, Peppelenbosch MP, van Deventer SJ. Mitogen activated protein (MAP) kinase signal transduction pathways and novel antiinflammatory targets. Gut. 2003;52(1):144-51.

56. Lo U, Selvaraj V, Plane JM, Chechneva OV, Otsu K, Deng W. p38a (MAPK14) critically regulates the immunological response and the production of specific cytokines and chemokines in astrocytes. Sci Rep. 2014;4:7405.

57. Jaeger S, Igea A, Arroyo R, Alcalde V, Canovas B. Orozco M Quantification of pathway cross-talk reveals novel synergistic drug combinations for breast cancer. Cancer Res. 2017;77(2):459-69.

58. Marone R, Cmiljanovic V, Giese B, Wymann MP. Targeting phosphoinositide 3-kinase-moving towards therapy. Biochim Biophys Acta. 2008;1784(1):159-85.

59. Yu JY, Ha JY, Kim KM, Jung YS, Jung JC, Oh S. Anti-Inflammatory activities of licorice extract and its active compounds, glycyrrhizic acid, liquiritin and liquiritigenin, in BV2 cells and mice liver. Molecules. 2015;20(7):13041-54.

60. Guan LP, Liu BY. Antidepressant-like effects and mechanisms of flavonoids and related analogues. Eur J Med Chem. 2016;121:47-57.

61. Yang HM, Wen L, Xu XY, Chang X, Liu YJ. Research progress of Chaihu Shugan Powder in the treatment of gastrointestinal diseases. Modern Medicine and Health Research. 2018;2(07):183.

62. He W. Advanced breast cancer treated with bupleurum power for soothing the liver combined with chemotherapy randomized parallel group study. J Practical Traditional Chinese Intern Med. 2014;28(8):83-6.

63. Shang, LZ, Ji S, Wang Q, Pan XL. Protection effect of bupleuri livercoursing powder on mammary gland hyperplasia induced by liver depression in rats. China Pharmacy. 2015;26(7):908-11. 